H. Omoto

Nagoya Math. J.

Vol. 70 (1978), 47-80

\title{
ON SINGULAR SETS OF FLAT HOLOMORPHIC MAPPINGS WITH ISOLATED SINGULARITIES
}

\author{
HIDEO OMOTO
}

\section{Introduction}

In [4] B. Iversen studied critical points of algebraic mappings, using algebraic-geometry methods. In particular when algebraic maps have only isolated singularities, he shows the following relation; Let $V$ and $S$ be compact connected non-singular algebraic varieties of $\operatorname{dim}_{C} V=n$, and $\operatorname{dim}_{C} S=1$, respectivelly. Suppose $f$ is an algebraic map of $V$ onto $S$ with isolated singularities. Then it follows that

$$
\chi(V)=(-1)^{n} \sum_{p} \mu_{f}(p)+\chi(S) \chi(F),
$$

where $\chi$ denotes the Euler number, $\mu_{f}(p)$ is the Milnor number of $f$ at the singular point $p$, and $F$ is the general fiber of $f: V \rightarrow S$.

The purpose of this paper is to generalize the above relation (0.1) as follows; Let $V$ and $W$ be connected compact complex manifolds of $\operatorname{dim}_{c}=n$, and $k$ respectively. And let $f$ be a flat holomorphic map of $V$ into $W$ with isolated singularities ([Def. 1.2]). Moreover we assume that rank $f \geqq k-1$. Then for generic points $p$ on singular set $\Sigma(f)$ of $f$ ([Def. 1.1]), we can define obstruction numbers $\mu_{f}(p) \in Z$ ([Def. 1.3]) associated with $f$ and $p$ which are Milnor numbers of $f$ at $p$ in the case $k=1$. However these numbers $\mu_{f}(p)$ are constant on irreducible component containing $p$ of $\Sigma_{\ell}(f)$. Therefore we put, with respect to, the irreducible decomposition $\Sigma(f)=\bigcup_{j=1} \Sigma^{(j)}$.

$$
\mu_{f}\left(\Sigma^{j}\right)=\mu_{f}(p) \quad \text { for any generic } p \in \Sigma^{(j)}
$$

Now our main theorem is to show the next relation; For the general fiber $F$ or $f: V \rightarrow W$,

$$
\chi(V)=(-1)^{n} \sum_{j=1}^{\ell} C\left(\mathscr{B}_{j}\right) \mu_{f}\left(\Sigma^{j}\right)+\chi(W) \chi(F)
$$

Received June 30, 1976. 
where $C\left(\mathscr{B}_{j}\right)$ are the Chern numbers of line bundles $\mathscr{B}_{j}$ over $\Sigma^{j}$ induced from $f(\operatorname{rank} f \geqq k-1)$.

In $\S 1$ we state some properties of singular set $\Sigma(f)$ ([3]) and give the definition of $\mu_{f}(p)$. In $\S 2$ we review differential geometrical definitions of connections, curvature and boundary forms ([2] and [6]). In $\S 3$ we show the duality formula of boundary forms which play an important role in proving the main theorem. The proof of the main theorem is done in $\S 4$.

\section{§1. Singularities of holomorphic maps}

1.1. Let $V$ be a complex manifold of complex dimension $n$ and $T^{*} V$ be the holomorphic cotangent bundle of $V$. Let $f$ be a holomorphic mapping of $V$ into a complex $k$-dimensional complex manifold $W(n \geqq k)$.

Definition 1.1. A point $p$ of $V$ is called a singular point of $f$, if $\operatorname{rank}_{p} f<k$, where $\operatorname{rank}_{p} f$ denotes the rank of the linear map $f_{p}^{*}: T_{f(p)}^{*} W$ $\rightarrow T_{p}^{*} V$. Moreover we denote by $\Sigma(f)$ the set of all singular points of $f$, called the singular set of $f$.

DEFINITION 1.2. $p \in \Sigma(f)$ is generic if the following conditions are satisfied;

i) $\operatorname{rank}_{p} f=k-1$,

ii) there exists a neighborhood $U$ of $p$ in $V$ such that $U \cap \Sigma(f)$ is a $(k-1)$-dimensional complex submanifold of $V$.

Let $p \in \Sigma(f)$ be generic. In order to define a topological number concerned with $p$, we take holomorphic coordinates $\left\{z^{i}\right\}_{i=1}^{n}$ on an open set $U_{p} \ni p$ in $V$ with $z^{i}(p)=0, i=1, \cdots, n$ and also local coordinates $\left\{w^{\alpha}\right\}_{\alpha=1}^{k}$ of $f(p)$ in $W$ such that $w^{\alpha}(f(p))=0, \alpha=1, \cdots, k$. Set $g^{\alpha}=w^{\alpha} \circ f$ and $g=\left(g^{1}, \cdots, g^{k}\right)$. Further let $\partial g / \partial z$ be the Jacobian matrix of $g$, that is,

$$
\frac{\partial g}{\partial z}=\left\|\begin{array}{l}
\frac{\partial g^{1}}{\partial z^{1}}, \cdots, \frac{\partial g^{k}}{\partial z^{1}} \\
\frac{\partial g^{1}}{\partial z^{n}}, \cdots, \frac{\partial g^{k}}{\partial z^{n}}
\end{array}\right\| .
$$

If we write $V(n, k ; C)$ for the Stiefel manifold consisting of all $k$-frames of $C^{n}$, then we have the holomorphic map 


$$
\frac{\partial g}{\partial z}: U_{p}-\Sigma(g) \rightarrow V(n, k ; C)
$$

As $p$ is generic, we can take a complex submanifold $\Sigma^{\perp}(p)$ as follows;

( $\alpha) \quad \Sigma \perp(p) \cap \Sigma(g)=\{p\}$.

( $\beta) \quad \Sigma^{\perp}(p)$ intersects transversally to $\Sigma(g)$ at $p$.

( $\gamma$ ) the boundary $\partial \Sigma^{\perp}(p)$ of $\Sigma^{\perp}(p)$ is a smooth manifold which is diffeomorphic to the $2(n-k)+1$-dimensional unit sphere $S^{2(n-k)+1}$ in $C^{2(n-k+1)}$.

We call the above submanifold $\Sigma^{\perp}(p)$ a complemental submanifold to $\Sigma(g)$ at $p$. Finally we choose $2(n-k)+1$-form $\omega_{n, k}$ on $V(n, k ; C)$ whose cohomology class $\hat{\omega}_{n, k}$ is the generator of $2(n-k)+1$ dimensional cohomology group of $V(n, k ; C), H^{2(n-k)+1}(V(n, k ; C) ; Z) \cong Z$. Here put

$$
\tilde{\mu}_{g}(p)=\int_{\partial \Sigma \perp(p)}\left(\frac{\partial g}{\partial z}\right)^{*} \omega_{n, k} .
$$

One notes that $\tilde{\mu}_{g}(p)$ is an integer and that $\tilde{\mu}_{g}(p)$ is independent of choosing local coordinates $\left\{z^{i}\right\}_{i=1}^{n},\left\{w^{\alpha}\right\}_{\alpha=1}^{k}$ and complemental submanifolds $\Sigma^{\perp}(p)$ to $\Sigma(g)$ at $p$. Therefore the following definition is well-defined.

DeFINITION 1.3. Let $p \in \Sigma(f)$ be generic. Then the obstruction number $\mu_{f}(p)$ at $p$ of $f$ is defined by

$$
\mu_{f}(p)=\tilde{\mu}_{g}(p) .
$$

1.2. Isolated singularities

We shall restrict our discussion to a holomorphic map $f: V \rightarrow W$ such that

i) $f$ has only isolated singularities, i.e., for any point $q \in W, f^{-1}(q)$ $\cap \Sigma(f)$ is an isolated points set in $V$.

ii) $f$ is flat.

For simplicity we call $f$ satisfying the above conditions i) and ii) an (IF)-holomorphic map. The following proposition is well-known.

Proposition 1.4. Let $f: V \rightarrow W$ be (IF)-holomorphic. Then the singular set $\Sigma(f)$ of $f$ is an analytic set of $V$ such that

$$
\operatorname{dim}_{C} \Sigma(f)=\operatorname{dim}_{C} W-1 .
$$

From now on we assume that $V$ and $W$ are connected compact complex manifolds of $\operatorname{dim}_{C} V=n$, and $\operatorname{dim}_{C} W=k$ and that $f: V \rightarrow W$ is (IF)-holomorphic such that $\operatorname{rank} f \geqq k-1$. 
LEMMA 1.5. Let $f: V \rightarrow W$ be as above and let $\Sigma(f)=\bigcup_{i=1}^{e} \Sigma^{(i)}$ be an irreducible decomposition of $\Sigma(f)$. Then for each $\Sigma^{(i)}$ the obstruction number $\mu_{f}\left(p_{i}\right)$ of $f$ at any generic point $p_{i} \in \Sigma^{(i)}$ is defined and constant.

Proof. It is clear from Proposition 1.4 and $\operatorname{rank} f \geqq k-1$ that generic points of $\Sigma(f)$ become regular points of the analytic set $\Sigma(f)$ in $V$, and conversely. Since the regular set of $\Sigma^{(i)}$ is connected, this lemma is trivial from the definition of the obstruction number. Q.E.D.

By the above lemma we can put $\mu_{f}\left(\Sigma^{(i)}\right)=\mu_{f}\left(p_{i}\right)$ for any generic point $p_{i} \in \Sigma^{(i)}$.

Next les us consider the following sequence concerning with $f: V$ $\rightarrow W$

$$
0 \longrightarrow \operatorname{Ker} T(f) \longrightarrow f^{\sharp}\left(T^{*} W\right) \stackrel{T(f)}{\longrightarrow} T^{*} V,
$$

where $f^{\sharp}\left(T^{*} W\right)$ is the induced bundle of $T^{*} W$ by $f$ and $T(f)$ is the linear map defined by

$$
T(f)(p, v)=f_{p}^{*} v \quad \text { for any }(p, v) \in f^{\sharp}\left(T^{*} W\right) .
$$

As $\operatorname{rank} f \geqq k-1\left(\operatorname{dim}_{c} W=k\right)$, we have

$$
(\operatorname{Ker} T(f))_{p}=\{0\} \quad \text { if } p \notin \Sigma(f)
$$

and

$$
\operatorname{dim}_{c}(\operatorname{Ker} T(f))_{p}=1 \quad \text { for } p \in \Sigma(f) .
$$

Thus the restricted bundle $\operatorname{Ker} T(f) \mid \Sigma(f)$ becomes a topological onedimensional complex vector bundle. Let us denote by $Q(\Sigma(f))$ the quotient bundle $f^{\sharp}\left(T^{*} W\right) / \operatorname{Ker} T(f) \mid \Sigma(f)$, and let $\sim: f^{\sharp}\left(T^{*} W\right) \mid \Sigma(f) \rightarrow Q(\Sigma(f))$ be the natural projection. Now let $p \in \Sigma(f)$ be generic. Suppose that $\omega$ is a type $(1,0)$-differential form defined on an open set $U \ni f(p)$ such that

$$
\text { zeros of } f^{*}(\omega) \cap f^{-1}(U)=p .
$$

Here let $f^{\sharp}(\omega)$ be the cross-section of $f^{\sharp}\left(T^{*} W\right)$ defined by

$$
f^{\sharp}(\omega)(p)=\left(p, \omega_{f(p)}\right) \quad \text { for any } p \in U .
$$

Then $\widetilde{f^{\#}(\omega)}$ is the continuous section of $Q(\Sigma(f))$ on $f^{-1}(U) \cap \Sigma(f)$, 
and from (1.2) we get

$$
\text { zeros of } \widetilde{f^{\#}}(\omega)=\{p\},
$$

that is, $f^{\sharp}(\omega)(p) \in \operatorname{Ker} T(f)_{p}$. Since $p$ is generic, there exists a neighborhood $U_{p}$ of $p$ in $\Sigma(f)$, which is a complex submanifold of $V$ of $\operatorname{dim}_{C}(k-1)$, included in $f^{-1}(U)$. Therefore $Q(\Sigma(f)) \mid U_{p}$ is a $(k-1)$ dimensional holomorphic vector bundle. Here we can define canonically the order of zeros $I_{p}\left(\widetilde{\left.f^{\#}(\omega)\right)}\right.$ of $\widetilde{f^{\#}(\omega)}$ at $p$.

DEFINITION 1.6. Let $p \in \Sigma(f)$ be generic and let $\omega$ be a $(1,0)$-type differential form on an open set $U \ni f(p)$ satisfying (1.2). Then the restricted index $\tilde{I}_{p}(\omega)$ of $\omega$ at $p$ is the order of zeros of $f^{\sharp}(\omega)$ at $p$, i.e.

$$
\tilde{I}_{p}(\omega)=I_{p}\left(f^{\#}(\omega)\right) .
$$

Before we state our theorem, we need

DeFinition 1.7. Let $f: V \rightarrow W$ be as before, we call $a(1,0)$-type differential form $\omega$ on $W$, an $f$-form, when the following conditions are satisfied ;

i) The zeros of $\omega$ is a finite points set such that

$$
f(\Sigma(f)) \cap z e r o s \text { of } \omega=\phi
$$

ii) $\Sigma(f) \cap$ zeros of $f^{*} \omega$ is also a finite points set whose points are all generic.

Let $\omega$ be an $f$-form. Then from the above condition ii) we can define the restricted index $\tilde{I}_{p}(\omega)$ for each $p \in \Sigma(f) \cap$ zeros of $f^{*} \omega$. The following existence proposition is proved by using the transversality theorem in [5].

Proposition 1.8. Let $V$ and $W$ be compact complex manifolds and let $f: V \rightarrow W$ be an (IF)-holomorphic map with $\operatorname{rank} f \geqq k-1,\left(\operatorname{dim}_{c} W=k\right)$. Then there exists an f-form $\omega$.

We shall prove this proposition in Appendix. Now we are in a position to state the following

THEOREM 1.9. Let $V$ and $W$ be connected compact complex manifolds of $\operatorname{dim}_{C} V=n$ and $\operatorname{dim}_{C} W=k$. Suppose that $f: V \rightarrow W$ is an (IF)holomorphic mapping such that $\operatorname{rank} f \geqq k-1$. Then if $F$ denotes a 
general fiber of $f: V \rightarrow W$, we have

$$
\chi(V)=\sum_{j=1}^{\ell}(-1)^{n} \tilde{I}_{p_{j}}(\omega) \mu_{f}\left(p_{j}\right)+\chi(W) \chi(F),
$$

where $\chi$ represents the Euler number and $\omega$ is an f-form such that $\Sigma(f) \cap$ zeros of $f^{*} \omega=\left\{p_{1}, \cdots, p_{\ell}\right\},(c f$. Def. 1.7).

Moreover we claim that (1.4) is independent of $f$-forms $\omega$.

MAIN THEOREM. Under assumptions in Theorem 1.9, let $\Sigma(f)=\bigcup_{i=1}^{r} \Sigma^{(i)}$ be the irreducible decomposition of the singular set $\Sigma(f)$ of $f$. Let $Q(f)=f^{\sharp}\left(T^{*} W\right) / \operatorname{Ker} T(f) \mid \Sigma(f)$ as in 1.1. Here put $\mathscr{B}_{i}=Q(f) \mid \Sigma^{(i)}$, $(i=1, \cdots, r)$. Then it follows that

$$
\chi(V)=(-1)^{n} \sum_{i=1}^{r} C\left(\mathscr{B}_{i}\right) \mu_{f}\left(\Sigma^{(i)}\right)+\chi(W) \chi(F),
$$

where $C\left(\mathscr{B}_{i}\right)$ denotes the topological $(k-1)$-th Chern number of the complex $(k-1)$-dimensional vector bundle $\mathscr{B}_{i}$ over $\Sigma^{(i)}$, in the sense of Steenrod [7].

Proof. Without loss of generality we can assume that $\Sigma(f)$ is irreducible, because any point of $\Sigma^{(i)} \cap \Sigma^{(j)}(i \neq j)$ is not generic. Then we have from Lemma 1.5 and (1.4)

$$
\chi(V)=(-1)^{n} \sum_{j=1}^{\ell} \tilde{I}_{p_{j}}(\omega) \mu_{f}(\Sigma(f))+\chi(W) \chi(F) .
$$

Since $\Sigma(f)$ is a compact analytic set of $V, \Sigma(f)$ becomes a compact $C W$ complex. Thus one can define the $(k-1)$-th chern number $C(Q(f))$ of $Q(f)$ in virtue of Steenrod. On the other hand, $\widetilde{f}^{\sharp}(\omega)$ is a continuous cross-section of $Q(f)$ and we see zero $\left(\widetilde{f^{*}}(\omega)\right)=\left\{p_{1}, \cdots, p_{\ell}\right\}$, here is the natural projection of $f^{\sharp}\left(T^{*} W\right) \mid \Sigma(f)$ onto $Q(f)$. The Steenrod's theorem shows that

$$
C(Q(f))=\sum_{j=1}^{\ell} I_{p_{j}}\left(\widetilde{f^{\sharp}}(\omega)\right)
$$

that is

$$
C(Q(f))=\sum_{j=1}^{\ell} \tilde{I}_{p_{j}}(\omega)
$$

Combining this fact with (1.6), we can prove (1.5).

Q.E.D. 
In particular in case of $k=1$, that is, $W$ is a compact Riemann surface, $\Sigma(f)$ is a finite points set, say $\left\{p_{1}, \cdots, p_{r}\right\}$ and $\mu_{f}\left(p_{j}\right)$ is the Milnor number of $f$ at the isolated singular point $p_{j}, j=1, \cdots, r$. Here from the main theorem we have

Corollary 1.10 [4]. Let $W$ be a connected compact Riemann surface and $f$ a holomorphic map of a connected compact complex manifold $V$ into $W$ with isolated singularities, say $\Sigma(f)=\left\{p_{1}, \cdots, p_{\ell}\right\}$. Then we get

$$
\chi(V)=(-1)^{n} \sum_{j=1}^{b} \mu_{f}\left(p_{j}\right)+\chi(W) \chi(F),
$$

where $F$ is a generic fibre of $f: V \rightarrow W$ and $n=\operatorname{dim}_{c} V$.

Proof. We know that $f$ is flat, because $\Sigma(f)$ is a finite points set and $\operatorname{dim}_{c} W=1$. Here the proof is trivial.

Q.E.D.

\section{§2. Connections and boundary forms ([2], [6])}

In this section we review several geometrical definitions in [2] and [6] to be used in the next section.

2.1. Let $V$ be a complex manifold and $A^{k}(V)$ the set of all $k$-forms on $V, k=1, \ldots, 2 m\left(\operatorname{dim}_{R} V=2 m\right)$. Let $E$ be a holomorphic vector bundle of fibre dimension $n$ over $V$ and $N$ be a hermitian norm on $E$. Here we denote by $\langle$,$\rangle the inner product induced by N$. Then we can define a canonical connection $D(N)$ on $E$ as in [2], as follows; Let $U$ be an open set of $V$ such that there exists a holomorphic frame $s=\left(s_{1}, \ldots\right.$, $s_{n}$ ), where $s_{i}$ is a holomorphic section of $E \mid U$. Put $N(s)=\left\|\left\langle s_{i}, s_{j}\right\rangle\right\|_{1 \leq i, j \leq n}$, and

$$
\theta=\theta(s, N)=d^{\prime} N(s) \cdot N(s)^{-1}, \quad \text { i.e., } \quad \theta_{i j}=\sum_{k=1}^{n} d^{\prime} N(s)_{i k} \cdot\left(N(s)^{-1}\right)_{k j},
$$

where $d^{\prime}$ is the type $(1,0)$-derivation. For a section $\xi=\sum_{k=1}^{n} \xi^{k} s_{k}$ of $E \mid U$, we define the covariant differential $D \xi=\sum_{k=1}^{n} d \xi^{k} \cdot s_{k}+\sum \xi^{k} D s_{k}$, where $D s_{k}=\sum_{j} \theta_{k j} s_{j}$. Then $D \xi$ is an $E$-valued 1-form and $d\langle\xi, \eta\rangle$ $=\langle D \xi, \eta\rangle+\langle\xi, D \eta\rangle$ for sections $\xi, \eta$ of $E$.

We call the above connection $D=D(N)$ the $N$-connection of $E$. Moreover the curvature form $K(s, D)=\left\|K_{i j}\right\|$ is given by

$$
K(s, D(N))=d \theta(s, N)-\theta(s, N) \wedge \theta(s, N) .
$$


$K(s, D(N))$ is called the curvature matrix of $D(N)$ relative to the frame $s$. Take another frame $s^{\prime}=\left(s_{1}^{\prime}, s_{n}^{\prime}\right)$ of $E \mid U$, and put $s_{i}^{\prime}=\sum_{j} A_{i j} \cdot s_{j}$. Then we get

$$
A K(s, D) A^{-1}=K\left(s^{\prime}, D\right) .
$$

Now we shall define the $k$-th chern form $C_{k}(E)$ of $E$ associated with the norm $N$. Let $M_{n}$ be the $n \times n$-complex matrices. Let $b_{k}^{n}: M_{n} \overbrace{\times \cdots \times}^{k} M_{n}$ $\rightarrow C$ be a $k$-linear map defined by

$$
b_{k}^{n}\left(A_{1}, \cdots, A_{k}\right)=\left.\frac{1}{k !} \frac{\partial^{k}}{\partial \lambda_{1} \cdots \partial \lambda_{k}}\right|_{\lambda_{1}=\cdots=\lambda_{k}=0} \operatorname{det}\left(1_{n}+\lambda_{1} A_{1}+\cdots+\lambda_{k} A_{k}\right)
$$

for $A_{i} \in M_{n}$, where $1_{n}$ denotes the unit matrix of degree $n$. For simplicity we set $b_{k}^{n}(A, \cdots, A)=b_{k}^{n}((A))$. Then it follows from (2.3) and the definition of $b_{k}^{n}$ that $b_{k}^{n}\left(\left(\frac{\sqrt{-1}}{2 \pi} K(s, D)\right)\right)$ is independent of the frame $s$.

Definition 2.1. Let $D=D(N)$ be an $N$-connection on $E$. Then the $k$-th Chern form $C_{k}(E, D)$ induced by $N$ is a type $(k, k)$-real form on $V$ defined as follows; for any frame $s$ of $E \mid U$ ( $s$ may be smooth),

$$
\left.C_{k}(E, D)\right|_{U}=b_{k}^{n}\left(\left(\frac{\sqrt{-1}}{2 \pi} K(s, D)\right)\right) \text {. }
$$

The next proposition is directly proved from the above definition ([1]).

Proposition 2.2. $C_{k}(E, D)$ is closed, i.e., $d C_{k}(E, D)=0$.

2.2. Duality formula and boundary form.

Suppose now that the following sequence $(\Sigma)$ of holomophic vector bundles over a complex manifold $V$ is exact,

$$
0 \rightarrow E_{\mathrm{I}} \rightarrow E \rightarrow E_{\mathrm{II}} \rightarrow 0 \text {. }
$$

If $E$ has a hermitian norm $N$, then in virtue of the sequence $(\Sigma)$ norms $N_{\mathrm{I}}$ and $N_{\mathrm{II}}$ are induced from $N$, on $E_{\mathrm{I}}$ and $E_{\mathrm{II}}$, respectively. Let $p_{\mathrm{I}}$ : $E \rightarrow E_{\mathrm{I}}$ and $p_{\mathrm{II}}: E \rightarrow E_{\mathrm{II}}$ be orthogonal projections and $D=D(N)$ be the $N$-connection on $E$. It follows then that $P_{i} D P_{i}=D_{i}$ becomes the $N_{i}$ connection on $E_{i}$ for $i=\mathrm{I}$, II. Moreover put $D_{t}=D+\left(e^{t}-1\right) P_{\mathrm{II}} D P_{\mathrm{I}}$ $(t \in R)$. $\quad D_{t}$ is also a connection on $E$, called $R$-family of $D$. The following proposition is used to define the boundary form of $E$. 
Duality formula [2]. Let all notations be as above. Snppose that $\operatorname{dim} E=n$ and $\operatorname{dim} E_{\mathrm{I}}=k$. Then it follows that

$$
\begin{aligned}
C_{n}(E, D)= & C_{k}\left(E_{\mathrm{I}}, D_{\mathrm{I}}\right) C_{n-k}\left(E_{\mathrm{II}}, D_{\mathrm{II}}\right) \\
& -\frac{1}{4 \pi} d\left\{\lim _{t \rightarrow-\infty} d^{c} \int_{t}^{0} b_{n}^{n}\left(\left(\frac{\sqrt{-1}}{2 \pi} K\left[E, D_{t}\right] ; \frac{\sqrt{-1}}{2 \pi} P_{\mathrm{I}}\right)\right) d t\right\}
\end{aligned}
$$

where $d^{c}=\sqrt{-1}\left(d^{\prime \prime}-d^{\prime}\right), b_{n}^{n}((A ; b))=b_{n}^{n}(B, \overbrace{A, \cdots}^{n-1}, A)+\cdots+b(A, \cdots$, $A, B)$ and $K\left[E, D_{t}\right]$ is the curvature element of $D_{t}$, defined as follows; for any frame $s$ of $E$,

$$
K\left[E, D_{t}\right](s)=K\left(s, D_{t}\right) .
$$

Now let $E$ be a holomorphic vector bundle with a norm $N$ over $V$ of fiber dimension $n$ and let $\pi$ be the projection of $E$ onto $V$. Set $E_{0}=\{v \in E ; v \neq 0\}$ and $\pi \mid E_{0}=\pi_{0}$. And let $\pi_{0}^{*}(E)$ be the induced bundle of $E$ by $\pi_{0}$. Then one defines naturally the induced norm $\pi_{0}^{*}(N)$ by $\pi_{0}$ and $N$. Let $L(E)=\bigcup_{e \in E_{0}}\{(e, C e)\}$ be the line bundle over $E_{0}$ and $Q(E)$ the quotient bundle of $\pi_{0}^{*}(E)$ by $L(E)$. Then clearly we get the following exact sequence;

$\Sigma(E)$,

$$
0 \rightarrow L(E) \rightarrow \pi_{0}^{*}(E) \rightarrow Q(E) \rightarrow 0,
$$

At first we have

Proposition 2.3. [6].

$$
C_{n}\left(\pi_{0}^{\sharp}(E), D\left(\pi_{0}^{\sharp} N\right)\right)=\pi_{0}^{*} C_{n}(E, D(N)) .
$$

Let $\Delta: E_{0} \rightarrow L(E)$ be the global holomorphic section defined by $\Delta(e)$ $=(e, e)$ for $e \in E_{0}$. Then we know

$$
C_{1}\left(L(E), D\left(N_{\mathrm{I}}\right)\right)=\frac{-1}{4 \pi} d d^{c} \log \left(\pi_{0}^{\sharp} N\right)(\Delta),
$$

where $N_{\mathrm{I}}$ is the norm on $L(E)$ defined by the sequence $\Sigma(E)$ and the norm $\pi_{0}^{\sharp}(N)$ on $\pi_{0}^{\sharp}(E)$ as before. Applying the duality formula (2.4) to the sequence $\Sigma(E)$, we obtain from (2.5) and (2.6)

$$
\begin{aligned}
\pi_{0}^{*} C_{n}(E, D(N))= & \frac{-1}{4 \pi} d\left\{d^{c} \log \pi_{0}^{\sharp} N(\Delta) \cdot C_{n-1}\left(Q(E), D\left(N_{\mathrm{II}}\right)\right)\right. \\
& \left.+\lim _{t \rightarrow-\infty} d^{c} \int_{t}^{0} b_{n}^{n}\left(\left(\kappa K\left[\pi_{0}^{\sharp} E, D_{t}\left(\pi_{0}^{\sharp} N\right)\right] ; \kappa P_{\mathrm{I}}\right)\right) d t\right\},
\end{aligned}
$$


where $\kappa=\frac{\sqrt{-1}}{2 \pi}$.

Here let us put

$$
\begin{aligned}
\eta_{n}(E, N, \Delta)= & \frac{-1}{4 \pi}\left\{d^{c} \log \pi_{0}^{\#} N(\Delta) \cdot C_{n-1}(Q(E))\right. \\
& \left.+\lim _{t \rightarrow-\infty} d^{c} \int_{t}^{0} b_{n}^{n}\left(\left(\kappa K\left[\pi_{0}^{\sharp} E, D_{t}\left(\pi_{0}^{\sharp} N\right)\right] ; \kappa P_{\mathrm{I}}\right)\right)\right\} .
\end{aligned}
$$

It is trivial

$$
d \eta_{n}(E, N, \Delta)=\pi_{0}^{*} C_{n}(E, D(N))
$$

We resume the above discussions as the following

DEFINITION 2.4 [6]. Let $E$ be a holomorphic $n$-dimensional vector bundle over a complex manifold $V$ with a norm $N$. Then the $(2 n-1)$ form $\eta_{n}(E, N, \Delta)$ on $E_{0}$ defined by (2.7) is called the boundary form of $E$ with the norm $N$.

For simplicity we abbreviate $\eta_{n}(E, N, \Delta)$ to $\eta_{n}(E)$ or $\eta_{n}(E, N)$.

\section{§3. Duality formula of boundary forms}

Let $V$ be a complex manifold and $A^{k}(V)$ the $k$-forms on $V$. Assume there exists an exact sequence $(\Sigma)$ of holomorphic vector bundles over $V$

$$
0 \rightarrow E_{\mathrm{I}} \rightarrow E \rightarrow E_{\mathrm{II}} \rightarrow 0 \text {. }
$$

Put $\left(E_{\mathrm{I}}\right)_{0}=\left\{v \in E_{\mathrm{I}} ; v \neq 0\right\}$ and let $\prod_{\mathrm{I}}$ be the projection of $\left(E_{\mathrm{I}}\right)_{0}$ onto $V$. Moreover let $\iota:\left(E_{\mathrm{I}}\right)_{0} \rightarrow E$ be the inclusion linear map in $(\Sigma)$. The purpose of this section is to prove the following

MAIN Lemma. All notations are as in $\S 2$. Let $\operatorname{dim} E_{\mathrm{I}}=k$ and $\operatorname{dim} E=n$, and let $N$ be a norm on $E$. Then it follows that, on $\left(E_{\mathrm{I}}\right)_{0}$

$$
\iota^{*} \eta_{n}(E)=\eta_{k}\left(E_{\mathrm{I}}\right) \prod_{1}^{*} C_{n-k}\left(E_{\mathrm{II}}\right)+\prod_{\mathrm{I}}^{*} \xi+d \Phi,
$$

where $\xi \in A^{2 n-1}(V)$ and $\Phi \in A^{2 n-2}\left(\left(E_{1}\right)_{0}\right)$.

3.1. In order to show (3.1) we need several lemmas. At first we have the following

LEMMA 3.1. There exists an element $\xi^{\prime} \in A^{2 n-1}(V)$ satisfying the following condition; Let us put 


$$
\psi=\iota^{*} \eta_{n}(E)-\eta_{k}\left(E_{\mathrm{I}}\right) \prod_{\mathrm{I}}^{*} C_{n-k}\left(E_{\mathrm{II}}\right)+\prod_{\mathrm{I}}^{*} \xi^{\prime}
$$

Then $\psi$ is closed, that is, $d \psi=0$.

Proof. Let $\prod_{0}: E_{0} \rightarrow V$ be the natural projection as before $\left(E_{0} \subset E\right)$. It is clear by (2.8) that

$$
\begin{aligned}
d\left(\iota^{*} \eta_{n}(E)\right. & \left.-\eta_{k}\left(E_{\mathrm{I}}\right) \prod_{\mathrm{I}}^{*} C_{n-k}\left(E_{\mathrm{II}}\right)\right) \\
& =\iota^{*} \prod_{0}^{*} C_{n}(E)-\prod_{\mathrm{I}}^{*} C_{k}\left(E_{\mathrm{I}}\right) \prod_{\mathrm{I}}^{*} C_{n-k}\left(E_{\mathrm{II}}\right) \\
& =\prod_{\mathrm{I}}^{*}\left\{C_{n}(E)-C_{k}\left(E_{\mathrm{I}}\right) C_{n-k}\left(E_{\mathrm{II}}\right)\right\} .
\end{aligned}
$$

Here we get $\xi^{\prime \prime} \in A^{2 n-1}(V)$ by the duality formula in $\S 2$ such that

$$
C_{n}(E)-C_{k}\left(E_{\mathrm{I}}\right) C_{n-k}\left(E_{\mathrm{II}}\right)=d \xi^{\prime \prime} \text {. }
$$

Thus putting $\xi^{\prime}=-\xi^{\prime \prime}$, we can prove (3.2).

Q.E.D.

Next let $S\left(E_{\mathrm{I}}\right)$ be the sphere bundle of $E_{\mathrm{I}}$, that is, $S\left(E_{\mathrm{I}}\right)=\left\{v \in E_{\mathrm{I}}\right.$; $\left.N_{\mathrm{I}}(v)=1\right\}$, and let $P: S\left(E_{\mathrm{I}}\right) \rightarrow V$ be the projection. Clearly each fibre of $S\left(E_{\mathrm{l}}\right)$ has the canonical orientation. One can here define the fibre integral $P_{*}: A^{\ell}\left(S\left(E_{\mathrm{I}}\right)\right) \rightarrow A^{\ell-(2 k-1)}(V)$ as follows; for any $\theta \in A^{\ell}\left(S\left(E_{\mathrm{I}}\right)\right)$,

$$
P_{*}(\theta)_{x}=\int_{P^{-1}(x)} \theta \quad(x \in V) .
$$

The following lemma is proved in Hirzebruch-Borel [1].

LEMMA 3.2 [4]. There is $\omega \in A^{2 k-1}\left(S\left(E_{\mathrm{I}}\right)\right.$ ) such that $d \omega=0$ and $P_{*}(\omega)=1$. Moreover for any closed form $\theta \in A^{\ell}\left(S\left(E_{\mathrm{I}}\right)\right)$ we write $\hat{\theta}$ the cohomology class of $\theta$. Then it follows that

$$
\hat{\theta}=\widehat{P^{*}\left(P_{*}(\theta)\right) \wedge \omega}+P^{*}\left(\xi_{1}\right)
$$

where $\xi_{1} \in H^{\ell}(V ; R)$.

Proposition 3.3. Let $\iota_{s}: S\left(E_{\mathrm{I}}\right) \rightarrow\left(E_{\mathrm{I}}\right)_{0}$ be the inclusion map and let $\psi$ be the $(2 n-1)$-form on $\left(E_{1}\right)_{0}$ as in Lemma 3.1. Then we obtain

$$
\widehat{P_{*}\left(\iota_{s}^{*} \psi\right)}=0 \text {. }
$$

If (3.3) is proved, we get our main lemma as Corollary. Indeed it follows from Lemma 3.2 that

$$
\widehat{\iota_{s}^{*}(\psi)}=\widehat{P^{*}\left(P_{*}\left(\iota_{s}^{*} \psi\right)\right)} \wedge \omega+P^{*}\left(\hat{\xi}_{1}\right)
$$

where $\xi_{1}$ is a $(2 n-1)$-closed form on $V$. Therefore we have from (3.3) 


$$
\widehat{\iota_{s}^{*}(\psi)}-\widehat{P^{*}\left(\xi_{1}\right)}=0
$$

Set here $\varphi=\psi-\prod_{\mathrm{I}}^{*}\left(\xi_{\mathrm{I}}\right)$ ( $\prod_{\mathrm{I}}^{*}$ is the projection of $\left(E_{\mathrm{I}}\right)_{0}$ onto $V$ ). Noticing $P=\prod_{\mathrm{r}} \circ \iota_{s}$, it follows

$$
\widehat{\iota_{s}^{*} \varphi}=\widehat{\iota_{s}^{*} \psi}-\widehat{P^{*}\left(\xi_{1}\right)}=0
$$

However since $H^{2 n-1}\left(\left(E_{\mathrm{I}}\right)_{0} ; \boldsymbol{R}\right)$ is isomorphic to $H^{2 n-1}\left(S\left(E_{\mathrm{I}}\right) ; \boldsymbol{R}\right)$ by $\iota_{s}^{*}$, we have $\hat{\varphi}=0$ in $H^{2 n-1}\left(\left(E_{1}\right)_{0} ; R\right)$. Thus it follows $\varphi=\psi-\prod_{1}^{*}\left(\xi_{1}\right)=d \Phi$ for some $\Phi \in A^{2 n-2}\left(\left(E_{\mathrm{I}}\right)_{0}\right)$ and so (3.1) in main lemma is proved.

3.2. The proof of Proposition 3.3.

All notations in $\S 2$ are used in this paragraph.

Let $N$ be the norm on $E$ and let $N_{i}$ be the norm on $E_{i}$ induced by $N(i=\mathrm{I}, \mathrm{II})$ in terms of the sequence $(\Sigma): 0 \rightarrow E_{\mathrm{I}} \rightarrow E \rightarrow E_{\mathrm{II}} \rightarrow 0$. The inclusion map $\iota:\left(E_{\mathrm{I}}\right)_{0} \rightarrow E_{0}(\subset E)$ induces the canonical linear map of $\prod_{\mathrm{I}}^{\#}(E)$ into $\prod_{0}^{\#}(E)$, which we denote by $\iota$ without confusion. Note $\iota^{\#}\left(\prod_{0}^{\#}(E)\right)$ $=\prod_{I}^{\#}(E)$ and $\iota^{\#}(L(E))=L\left(E_{\mathrm{I}}\right)$. Then we have the following diagram

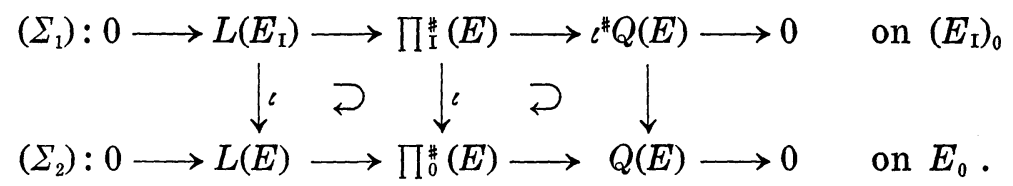

Remark. $\left(\Sigma_{1}\right)$ is the restriction of $\left(\Sigma_{2}\right)$ to $\left(E_{\mathrm{I}}\right)_{0}$ and $\Sigma_{i}$ is exact for $i=1,2$.

First of all, using Proposition 3.5 in [6], it follows from the exact sequence $\left(\Sigma_{2}\right)$ that

$$
\begin{aligned}
\iota^{*} \eta_{n}(E, N)= & -\frac{1}{4 \pi}\left\{d^{c} \log \prod_{\mathrm{I}}^{\#}\left(N_{\mathrm{I}}\right)(\Delta \circ \iota) \cdot \iota^{*} C_{n-1}(Q(E))\right. \\
& \left.\left.+\lim _{t \rightarrow-\infty} d^{c} \int_{t}^{0} b_{n}^{n}\left(C \kappa K\left[\prod_{\mathrm{I}}^{*}(E), D_{t}\left(\prod_{\mathrm{I}}^{\#} N\right)\right] ; \kappa P_{\mathrm{I}}\right)\right)\right\}
\end{aligned}
$$

where $\Delta: E_{0} \rightarrow L(E)$ is the section defined by

$$
\Delta(e)=(e, e) \quad \text { for } e \in E_{0} \text {. }
$$

Let us consider the form $\iota^{*} C_{n-1}(Q(E))$ in the left hand side of (3.4). For this purpose take the following commutative diagram over $\left(E_{\mathrm{I}}\right)_{0}$; 


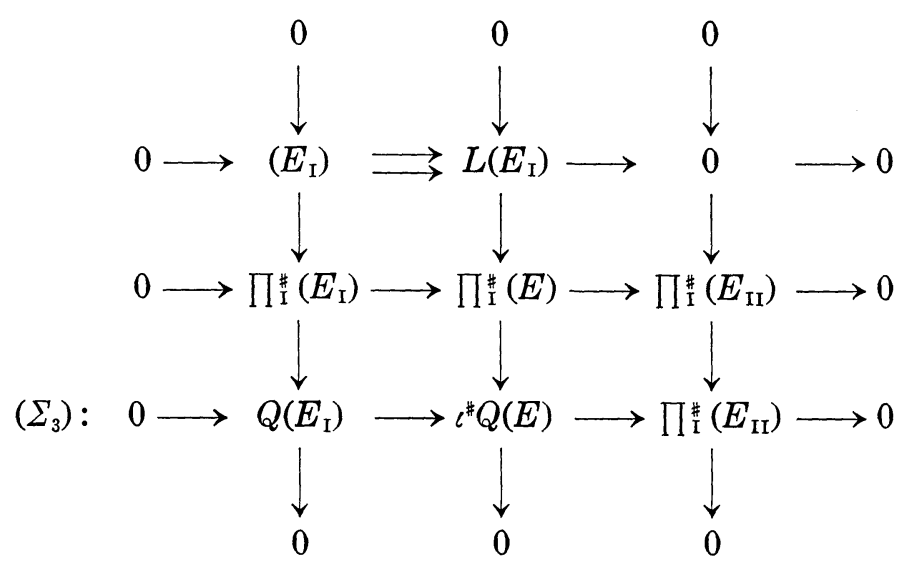

where each sequence is exact.

It is well-known (cf. (3.23) in [6] that

$$
\iota^{*} C_{n-1}(Q(E))=C_{n-1}\left(\iota^{\sharp} Q(E)\right) .
$$

Therefore using the exact sequence $\left(\Sigma_{3}\right)$ in the above diagram, we find from duality formula,

$$
\begin{aligned}
\iota^{*} C_{n-1}(Q(E))= & C_{k-1}\left(Q\left(E_{\mathrm{I}}\right)\right) \prod_{1}^{*} C_{n-k}\left(E_{\mathrm{II}}\right) \\
& -\frac{\pi}{4} d \lim _{t \rightarrow-\infty} d^{c} \int_{t}^{0} b_{n-1}^{n-1}\left(\left(\kappa K\left[\iota^{\sharp} Q(E), D_{t}^{Q}\right] ; \kappa P_{\mathrm{I}}^{Q}\right)\right) d t
\end{aligned}
$$

where $P_{\mathrm{I}}^{Q}: \iota^{\sharp} Q(E) \rightarrow Q\left(E_{\mathrm{I}}\right)$ is the orthogonal projection associated with $\left(\Sigma_{3}\right)$ and the norm $\iota^{\sharp}\left(N^{Q}\right)$ on $\iota^{\sharp} Q(E)$ induced by $N$, and $D_{t}^{Q}$ is $\boldsymbol{R}$-family of $D\left(\iota^{\sharp} N^{Q}\right)$ as in $\S 2$.

Furthermore, noting that $\Delta_{\circ} \iota:\left(E_{\mathrm{I}}\right)_{0} \rightarrow L\left(E_{\mathrm{I}}\right)$ is the canonical section for the boundary form $\eta_{k}\left(E_{\mathrm{I}}\right)$, we have from the first vertical exact sequence in the above diagram,

$$
\begin{aligned}
\eta_{k}\left(E_{\mathrm{I}}\right)= & -\frac{1}{4 \pi}\left\{d^{c} \log \prod_{\mathrm{I}}^{\#} N_{\mathrm{I}}(\Delta \circ \iota) C_{k-1}\left(Q\left(E_{\mathrm{I}}\right)\right)\right. \\
& \left.+\lim _{t \rightarrow-\infty} d^{c} \int_{t}^{0} b_{k}^{k}\left(\left(\kappa K\left[\prod_{\mathrm{I}}^{\#} E, D_{t}\left(\prod_{\mathrm{I}}^{\#} N_{\mathrm{I}}\right)\right] ; \kappa P_{\mathrm{I}}^{E \mathrm{I}}\right)\right) d t\right\},
\end{aligned}
$$

where $P_{\mathrm{I}}^{E_{\mathrm{I}}}: \prod_{\mathrm{I}}^{\#}\left(E_{\mathrm{I}}\right) \rightarrow L\left(E_{\mathrm{I}}\right)$ is the orthogonal projection.

Here it follows from (3.4), (3.5) and (3.6) that 


$$
\begin{aligned}
\iota^{*} \eta_{n}(E)- & \eta_{k}\left(E_{\mathrm{I}}\right) \prod_{\mathrm{I}}^{*} C_{n-k}\left(E_{\mathrm{II}}\right) \\
= & \text { const. } d^{c} \log \prod_{\mathrm{I}}^{\#} N_{\mathrm{I}}(\Delta \circ \iota) \cdot d \lim _{t \rightarrow-\infty} d^{c} \int_{t}^{0} b_{n-1}^{n-1}\left(\left(K\left[\iota^{\sharp} Q(E), D_{t}^{Q}\right] ; P_{\mathrm{I}}^{Q}\right)\right) d t \\
& + \text { const. } \prod_{\mathrm{I}}^{*} C_{n-k}\left(E_{\mathrm{II}}\right) \\
& \quad \prod_{\mathrm{I}}^{*} C_{n-k}\left(E_{\mathrm{II}}\right) \lim _{t \rightarrow-\infty} d^{c} \int_{t}^{\infty} b_{k}^{k}\left(\left(K\left[\prod_{\mathrm{I}}^{\#} E_{\mathrm{I}}, D_{t}\left(\prod_{\mathrm{I}}^{\#} N\right)\right] ; P_{\mathrm{I}}^{E_{\mathrm{I}}}\right)\right) d t \\
& + \text { const. } \lim _{t \rightarrow-\infty} d^{c} \int_{t}^{0} b_{n}^{n}\left(\left(K\left[\prod_{\mathrm{I}}^{\#} E, D_{t}\left(\prod_{\mathrm{I}}^{\#} N\right)\right] ; P_{\mathrm{I}}\right)\right) d t,
\end{aligned}
$$

(note $P_{\mathrm{I}}$ is the orthogonal projection of $E$ onto $E_{\mathrm{I}}$ in the sequence $\Sigma)$.

We shall show next that the first term of the right hand side of (3.7) has zero fibre integral, that is, if $P_{*}$ denotes the fibre integral of the sphere bundle $S\left(E_{\mathrm{I}}\right)$ of $E_{\mathrm{I}}$, then

$$
P_{*}\left(d^{c} \log \prod_{\frac{1}{1}}^{\sharp} N_{\mathrm{I}}(\Delta \circ \iota) \cdot d \lim _{t \rightarrow-\infty} d^{c} \int_{t}^{0} b_{n-1}^{n-1}\left(\left(K\left[\iota^{\sharp} Q(E), D_{t}^{Q}\right] ; P_{\mathrm{I}}^{Q}\right)\right) d t=0 ，\right.
$$

where $\iota_{s}:\left(E_{\mathrm{I}}\right)_{0} \rightarrow S\left(E_{\mathrm{I}}\right)$ is the inclusion.

For this aim let $1 \leq i, j \leq n, 1 \leq \alpha, \beta \leq k$, and $1 \leq A, B \leq n-k$. In the sequence $(\Sigma): 0 \rightarrow E_{\mathrm{I}} \rightarrow E \rightarrow E_{\mathrm{II}} \rightarrow 0, n$ and $k$ are dimensions of $E$ and $E_{\mathrm{I}}$, respectively, and $N$ is the norm on $E$ and $N_{i}$ denotes the induced norm on $E_{i}$ defined by $(\Sigma)$ and $N, i=\mathrm{I}$, II.

Now let $\left\{\tilde{e}_{i}\right\}_{i=1}^{n}$ be an orthonormal frame of $E$ over an open set $\tilde{U} \subset V$ such that

$$
\left\{\tilde{e}_{\alpha}\right\}_{\alpha=1}^{k} \text { is the orthonormal frame of } E \mid \tilde{U} \text {. }
$$

If $D=D(N)$ is the $N$-connection of $E$, one finds

$$
D \tilde{e}_{i}=\Sigma_{j} \tilde{\theta}_{i j} \tilde{e}_{j}, \quad \tilde{\theta}_{i j} \in A^{1}(\tilde{U}) .
$$

Let $\prod_{\mathrm{I}}:\left(E_{\mathrm{I}}\right)_{0} \rightarrow V$ be the natural projection and put $\Pi_{\mathrm{I}}^{-1}(\tilde{U})=U$. Since $\left\{\tilde{e}_{\alpha}\right\}$ is the frame of $E_{\mathrm{I}} \mid \tilde{U}$, we find $U \cong \tilde{U} \times C^{k}-\{0\}$ (diffeomorphic). Let us denote the canonical coordinates of $C^{k}$ by $\left\{z^{\alpha}\right\}_{\alpha=1}^{k}$.

Clearly $\left\{\tilde{e}_{i} \circ \prod_{1}\right\}_{i=1}^{n}$ becomes the orthonormal frame of $\prod_{1}^{\#}(E) \mid U$, with respect to the induced norm $\prod_{1}^{\#}(N)$. Putting $\tilde{e}_{i} \circ \prod_{i}=e_{i}(i=1, \cdots, n)$ we get

$$
D\left(\prod \frac{\#}{\mathrm{I}} N\right) e_{i}=\Sigma_{j} \theta_{i j} e_{j}, \quad \theta_{i j} \in A^{1}(U)
$$

where $\theta_{i j}=\prod_{\mathrm{I}}^{*}\left(\tilde{\theta}_{i j}\right)$. 
On the other hand for $\Delta \circ$, , we find

$$
\Delta \circ \iota\left(x, z^{1}, \cdots, z^{k}\right)=\Sigma_{\alpha} z^{\alpha} e_{\alpha}(x) \quad \text { for }\left(x, z^{1}, \cdots, z^{k}\right) \in \tilde{U} \times C^{k}-\{0\} .
$$

Let here $u_{1}$ be the smooth-section of $L\left(E_{\mathrm{I}}\right)$ defined by

$$
\begin{aligned}
u_{1}\left(x, z^{1}, \cdots, z^{k}\right) & =\left(\frac{\Delta \circ \iota}{\prod_{1}^{*} N_{\mathrm{I}}}\right)\left(x, z^{1}, \cdots, z^{k}\right) \\
& =\sum_{\alpha=1}^{k} \frac{z^{k}}{\sqrt{z^{1} z^{1}+\cdots+z^{k} z^{k}}} e_{\alpha}(x) .
\end{aligned}
$$

Set $U_{1}=\left\{\left(x, z^{1}, \ldots, z^{k}\right) ; z^{1} \neq 0\right\} \subset U$. Then we can choose another sections $u_{2}, \cdots, u_{k}$ of $\prod_{1}^{*}\left(E_{\mathrm{I}}\right) \mid U_{1}$ such that $\left\{u_{\alpha}\right\}_{\alpha=1}^{k}$ is the orthonormal frame. Thus we have

$$
\begin{cases}u_{\alpha}=\Sigma_{\beta} a_{\alpha \beta} e_{\beta} & a_{\alpha \beta} \in A^{0}\left(U_{1}\right) \\ e_{\beta}=\Sigma_{\alpha} b_{\beta \alpha} u_{\alpha} & b_{\alpha \beta} \in A^{0}\left(U_{1}\right)\end{cases}
$$

Then $a=\left\|a_{\alpha \beta}\right\|$, and $b=\left\|b_{\alpha \beta}\right\|$, are elements of the group of $k \times k$-unitary matrices $U(k)$ and $a=b^{-1}$. Here let us put

$$
\omega_{\alpha \beta}=\Sigma_{\gamma} d a_{\alpha \gamma} \cdot b_{\tau \beta} .
$$

For simplicity we consider $U(k)$ as the subspace of $n \times n$-matrices $M_{n}$ in the following way; $a \in U(k)$ corresponds to $\left(\frac{a}{0} \mid \frac{0}{0}\right) \in M_{n}$.

Now let $D^{\#}=D\left(\prod_{1}^{*} N\right)$ be the $\prod_{1}^{*} N$-connection of $\prod_{1}^{*} E$. Then it follows directly from (3.8) and (3.9) that with respect to the orthonormal frame $\left\{u_{1}, \cdots, u_{k}, e_{k+1}, \cdots, e_{n}\right\}$,

$$
\left\{\begin{array}{l}
D^{\sharp} u_{\alpha}=\sum_{\beta=1}^{k}\left\{\omega_{\alpha \beta}+(a \cdot \theta \cdot b)_{\alpha \beta}\right\} u_{\beta}+\sum_{A=1}^{n-k}(a \cdot \theta)_{\alpha, k+A} e_{k+A} \\
D^{\sharp} e_{k+A}=\sum_{\alpha}(\theta \cdot b)_{k+A}, u_{\alpha}+\sum_{B} \theta_{k+A, k+B} e_{k+B}
\end{array}\right.
$$

where $\theta=\left\|\theta_{i j}\right\|_{1 \leq i, j \leq n}$.

Next let $P_{\mathrm{II}}: \prod_{\mathrm{I}}^{*}(E) \rightarrow \iota^{\sharp} Q(E)$ be the orthogonal projection associated with the exact sequence: $0 \rightarrow L\left(E_{\mathrm{I}}\right) \rightarrow \prod_{\mathrm{I}}^{*}(E) \rightarrow \iota^{\sharp} Q(E) \rightarrow 0$. Remember that if we denote by $D^{Q}$ the canonical connection of $\iota^{\sharp} Q(E)$, then

$$
D^{Q}=P_{\mathrm{II}} D^{\sharp} P_{\mathrm{II}}
$$

and that $\left\{u_{2}, \cdots, u_{k}, e_{k+1}, \cdots, e_{n}\right\}$ is the orthonormal frame of $\iota^{\sharp} Q(E)$. Here let $2 \leq \tilde{\alpha}, \tilde{\beta} \leq k$. Then it follows from (3.10) that 


$$
\left\{\begin{array}{l}
D^{Q} u_{\tilde{\alpha}}=\sum_{\tilde{\beta}}\left\{\omega_{\tilde{\alpha} \tilde{\beta}}+(a \cdot \theta \cdot b)_{\tilde{\alpha} \tilde{\beta}}\right\} u_{\tilde{\beta}}+\sum_{A}(a \cdot \theta)_{\tilde{\alpha}, k+A} e_{k+A} \\
D^{Q} e_{k+A}=\sum_{\tilde{\alpha}}(\theta \cdot b)_{k+A, \tilde{\alpha}} u_{\tilde{\alpha}}+\sum_{B} \theta_{k+A, k+B} e_{k+B} .
\end{array}\right.
$$

For calculations of $b_{n-1}^{n-1}\left(\left(K\left[i^{\sharp} Q(E), D_{t}^{Q}\right] ; P_{1}^{Q}\right)\right)$ recall that $D_{t}^{Q}=D^{Q}$ $+\left(e^{t}-1\right) P_{\mathrm{II}}^{Q} D^{Q} P_{\mathrm{I}}^{Q}$, where $P_{\mathrm{I}}^{Q}: \iota^{\sharp} Q(E) \rightarrow Q\left(E_{\mathrm{I}}\right)$ and $P_{\mathrm{II}}^{Q}: \iota^{\sharp} Q(E) \rightarrow \prod_{\mathrm{I}}^{\#}\left(E_{\mathrm{II}}\right)$ are the orthogonal projections induced from the exact sequence

$$
0 \longrightarrow Q\left(E_{\mathrm{I}}\right) \longrightarrow \iota^{\#} Q(E) \longrightarrow \prod_{\mathrm{I}}^{\#}\left(E_{\mathrm{II}}\right) \longrightarrow 0 \text {. }
$$

Let $K\left(D_{t}^{Q}\right)$ be the curvature matrix of $D_{t}^{Q}$ with respect to the above frame $\left\{u_{2}, \cdots, u_{k}, e_{k+1}, \cdots, e_{n}\right\}$ of $\iota^{\sharp} Q(E)$ and put

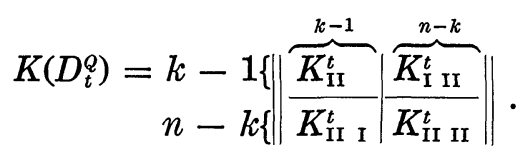

Then we get (cf. Lemma 4.8 [1])

$$
\left\{\begin{array}{l}
K_{\mathrm{II}}^{t}=K\left[Q\left(E_{\mathrm{I}}\right), P_{\mathrm{I}}^{Q} D^{Q} P_{\mathrm{I}}^{Q}\right]+e^{t} \square_{\mathrm{I}} \\
K_{\mathrm{II} \mathrm{II}}^{t}=K\left[\prod_{\mathrm{I}}^{\#}\left(E^{\mathrm{II}}\right), P_{\mathrm{II}}^{Q} D^{Q} P_{\mathrm{II}}^{Q}\right]+e^{t} \square_{\mathrm{II}} \\
K_{\mathrm{III}}^{t}=e^{t} P_{\mathrm{II}}\left(D^{Q}\right)^{2} P_{\mathrm{I}}, K_{\mathrm{II} \mathrm{I}}^{t}=e^{t} P_{\mathrm{I}}\left(D^{Q}\right)^{2} P_{\mathrm{II}}
\end{array}\right.
$$

where

$$
\square_{\mathrm{I}}=P_{\mathrm{I}}^{Q} D^{Q} P_{\mathrm{II}}^{Q} D^{Q} P_{\mathrm{I}}^{Q}
$$

and

$$
\square_{\mathrm{II}}=P_{\mathrm{II}}^{Q} D^{Q} P_{\mathrm{I}}^{Q} D^{Q} P_{\mathrm{II}}^{Q} \text {. }
$$

Remark. It is clear from the choice of the frame $\left\{u_{2}, \cdots, u_{k}, e_{k+1}\right.$, $\left.\cdots, e_{n}\right\}$ that

$$
\left\{\begin{array}{l}
P_{\mathrm{I}}^{Q} u_{\tilde{\alpha}}=u_{\tilde{\alpha}}, \quad P_{\mathrm{I}}^{Q} e_{k+A}=0 \\
P_{\mathrm{II}}^{Q} u_{\tilde{\alpha}}=0 \text { and } P_{\mathrm{II}}^{Q} e_{k+A}=e_{k+A}
\end{array}\right.
$$

Using this remark and (3.11), we shall compute each term in (3.12). At first we introduce the following notations. In general let $P$ be a differential fibre bundle over a differential manifold $M$, and $\Pi$ be the projection. Let $\omega$ be any differential form on $P$. We say that $\omega$ is at most of $k$-fibre degree, denoted by $F(\omega) \leq k$, when the following condition is satisfied; Let $y$ be any point of $P$ and $F_{y}$ be the fibre space passing through $y$. Then for any $k+1$ vectors $X_{1}, \cdots, X_{k+1} \in T_{y}\left(F_{y}\right)$ the 
inner derivative $\left.X_{1} \wedge \cdots \wedge X_{k+1}\right\rfloor \omega$ of $\omega$ with respect to $X_{1}, \cdots, X_{k+1}$ is zero, i.e., $\left.X_{1} \wedge \cdots \wedge X_{k+1}\right\lrcorner \omega=0$.

Under this notation each term in the right hand side of (3.12) is calculated as below.

a) $K\left[Q\left(E_{\mathrm{I}}\right), P_{\mathrm{I}}^{Q} D^{Q} P_{\mathrm{I}}^{Q}\right]=\left\|\omega_{\tilde{\alpha} 1} \wedge \omega_{1 \tilde{\beta}}\right\|_{2 \leq \tilde{\alpha}, \tilde{\beta} \leq k}+\Phi_{1}$, where $F\left(\Phi_{1}\right) \leq 1$.

Proof. First of all find from (3.11)

$$
P_{1}^{Q} D^{Q} u_{\tilde{\alpha}}=\sum_{\tilde{\beta}}\left\{\omega_{\tilde{\alpha} \tilde{\beta}}+(a \cdot \theta \cdot b)_{\tilde{\alpha} \tilde{\beta}}\right\} u_{\tilde{\beta}}
$$

Thus

$$
K\left[Q\left(E_{\mathrm{I}}\right), P_{\mathrm{I}}^{Q} D^{Q} P_{\mathrm{I}}^{Q}\right]=\left\|d \omega_{\tilde{\alpha} \tilde{\beta}}-\sum_{\tilde{\gamma}} \omega_{\tilde{\alpha} \tilde{\gamma}} \wedge \omega_{\tilde{\gamma} \tilde{\beta}}\right\|+\Phi_{1}
$$

where

$$
\begin{aligned}
\Phi_{1}= & \| d(a \cdot \theta \cdot b)_{\tilde{\alpha} \tilde{\beta}}-\sum_{\bar{\gamma}}(a \cdot \theta \cdot b)_{\tilde{\alpha} \tilde{\gamma}} \wedge\left(\omega_{\tilde{\gamma} \tilde{\beta}}+(a \cdot \theta \cdot b)_{\bar{\gamma} \tilde{\beta}}\right) \\
& -\sum_{\bar{\gamma}}\left(\omega_{\tilde{\alpha} \tilde{\gamma}}+(a \cdot \theta \cdot b)_{\tilde{\alpha} \tilde{\gamma}}\right) \wedge(\alpha \cdot \theta \cdot b)_{\tilde{\beta} \tilde{\gamma}} \| .
\end{aligned}
$$

However as $d \omega_{\alpha \beta}-\sum_{r=1}^{k} \omega_{\alpha \gamma} \wedge \omega_{r \beta}=0$, we get

$$
K\left[Q\left(E_{\mathrm{I}}\right), P_{\mathrm{I}}^{Q} D^{Q} P_{\mathrm{I}}^{Q}\right]=\left\|\omega_{\tilde{\alpha} 1} \wedge \omega_{1 \tilde{\beta}}\right\|+\Phi_{1} .
$$

The fact that $\Phi_{1}$ is at most fibre degree one is shown from $\theta=\prod_{1}^{*}(\tilde{\theta})$ (note $\left.\prod_{\mathrm{I}}:\left(E_{\mathrm{I}}\right)_{0} \rightarrow V\right)$.

b) $K\left[\prod_{\mathrm{I}}^{\#} E_{\mathrm{II}}, P_{\mathrm{II}}^{Q} D^{Q} P_{\mathrm{II}}^{Q}\right]=\left\|d \theta_{k+A, k+B}-\sum_{C} \theta_{k+A, k+C} \wedge \theta_{k+C, k+B}\right\|_{A, B}$.

This is trivial.

c) $F\left(\square_{\mathrm{I}}\right)=0$.

Proof. As $P_{\mathrm{II}}^{\mathrm{Q}} D^{Q} u_{\tilde{\alpha}}=\sum_{A}(a \cdot \theta)_{\tilde{\alpha}, k+A} e_{k+A}$, we have

$$
\begin{aligned}
P_{\mathrm{I}}^{Q} D^{Q} P_{\mathrm{II}} D^{Q} u_{\tilde{\alpha}} & =\sum_{A}(a \cdot \theta)_{\tilde{\alpha}, k+A} P_{\mathrm{I}}^{Q} D^{Q} e_{k+A} \\
& =\sum_{A, \tilde{\beta}}(a \cdot \theta)_{\tilde{\alpha}, k+A}(\theta \cdot b)_{k+A, \tilde{\beta}} u_{\tilde{\beta}} .
\end{aligned}
$$

This fact shows that $F\left(\square_{\mathrm{I}}\right)=0$.

d) $\square$ II $=-\left\|\sum_{\beta} \theta_{k+A, \beta} \theta_{\beta, k+\beta}\right\|+\left\|\sum_{\beta, \gamma} \theta_{k+A, \beta} a_{1 \gamma} \bar{a}_{1 \beta} \theta_{r, k+B}\right\|$.

Proof. Direct calculations show that 


$$
\begin{aligned}
\square_{\mathrm{II}} & =\left\|-\sum_{\tilde{\alpha}}(\theta \cdot b)_{k+A, \tilde{\alpha}} \wedge(a \cdot \theta)_{\tilde{\alpha}, k+B}\right\|_{A, B} \\
& =-\left\|\sum_{\tilde{\alpha} \tilde{\alpha} \gamma} \theta_{k+A, \beta} b_{\beta \tilde{\alpha}} a_{\alpha \tilde{\gamma}} \theta_{r, k+B}\right\| \\
& =-\left\|\sum_{\beta r} \theta_{k+A}\left(\delta_{\beta \gamma}-b_{\beta 1} a_{1 \gamma}\right) \theta_{r, k+B}\right\| \\
& =-\left\|\sum_{\beta} \theta_{k+A, \beta} \wedge \theta_{\beta, k+B}\right\|+\left\|\sum_{\beta r} \theta_{k+A, \gamma} a_{1 \beta} \bar{a}_{1 \beta} \theta_{r, k+B}\right\| .
\end{aligned}
$$

here we used ${ }^{t} \bar{a}=a^{-1}=b$ in the third equality.

Q.E.D.

e) $P_{\mathrm{II}}^{Q}\left(D^{Q}\right)^{2} P_{\mathrm{I}}^{Q}=\left\|\sum_{\gamma} \omega_{\tilde{\alpha} 1} a_{1 \gamma} \theta_{r, k+A}\right\|_{\tilde{\alpha}, A}+\Phi_{0}$

where $F\left(\Phi_{0}\right)=0$.

f) $P_{\mathrm{I}}^{Q}\left(D^{Q}\right)^{2} P_{\mathrm{II}}=-{ }^{t}\left\|\bar{\omega}_{\tilde{\alpha} 1} \bar{a}_{1 \gamma} \bar{\theta}_{r, k+A}\right\|+\Phi_{0}^{\prime}$

where ${ }^{t}\|\cdot\|$ denotes the transpose of the matrix $\|\cdot\|$ and $F\left(\Phi_{0}^{\prime}\right)=0$.

Since we can obtain e) and f) by the same computation before, we omit these calculations.

Hence it follows from a) $\sim$ f) that, with respect to the frame $\left\{u_{2}, \cdots\right.$, $\left.u_{k}, e_{k+1}, \cdots, e_{n}\right\}$ of $i^{\sharp} Q(E)$,

(3.13) $K\left[i^{\sharp} Q(E), D_{t}^{Q}\right]$

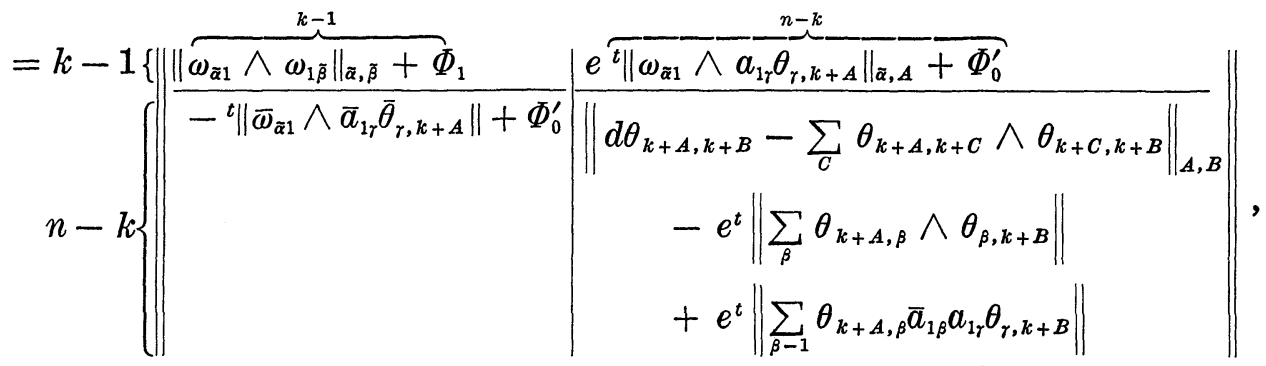

where $F\left(\Phi_{1}\right) \leq 1$ and $F\left(\Phi_{0}\right)=F\left(\Phi_{0}^{\prime}\right)=0$.

On the other hand for $P_{I}^{Q}$ we get

$$
P_{\mathrm{I}}^{Q}=\left\|\frac{\mathbf{1}_{k+1}}{0} \mid \frac{0}{0}\right\|,
$$

with respect to $\left\{u_{2}, \cdots, u_{k}, e_{k+1}, \cdots, e_{n}\right\}$.

finally we need the following elementary

Lemma 3.4. Let $A \in M_{n}$ and let $\Delta_{i j}(A)$ be the $(i, j)$-cofactor of $A$. Then one has

$$
b_{n}^{n}\left(\left(A ;\left(\frac{\mathbf{1}_{k}}{0} \mid \frac{0}{0}\right)\right)\right)=\sum_{j=1}^{k} \Delta_{j j}(A) .
$$


Proof. From the definition of $b_{n}^{n}((A ; B))$ in $\S 2$ this lemma is trivial.

Now applying Lemma 3.4 to (3.13) and (3.14), we have

Q.E.D.

$$
b_{n-1}^{n-1}\left(\left(K\left[\iota^{\sharp} Q(E), D_{t}^{Q}\right] ; P_{1}^{Q}\right)\right)=\sum_{i=1}^{k-1} \Delta_{i \imath}\left(K\left[\iota^{\sharp} Q(E), D_{t}^{Q}\right]\right) .
$$

Thus from (3.13) it follows that any term in $\Delta_{i i}\left(K\left[\iota^{\sharp} Q(E) ; D_{t}^{Q}\right]\right)(i=1, \ldots$, $k-1)$ is of type

$$
\begin{array}{ll}
\tilde{\alpha} & \tilde{\alpha} \\
\vee & \vee
\end{array}
$$

where $\Phi \in A^{0}(\tilde{U}), F\left(\Phi_{2 k-3}\right) \leq 2 k-3$ and $2 \leq \tilde{\gamma}_{j}, \tilde{\beta}_{j} \leq k,(j=1, \cdots, t)$.

Let us here represent $a_{1 \alpha}$ using the coordinates $\left\{z^{1}, \cdots, z^{k}\right\}$ of $C^{k}$. From (3.9), $u_{1}=\sum_{\alpha=1}^{k} a_{1 \alpha} e_{\alpha}$. But as $u_{1}=\sum \frac{z^{\alpha}}{\alpha \sqrt{\frac{z_{1} \bar{z}_{1}+\cdots+z_{k} \bar{z}_{k}}{2}}} e_{\alpha}$ it follows that

$$
a_{1 \alpha}=\frac{z^{\alpha}}{|z|}, \quad\left(|z|=\sqrt{\left.z^{1} \bar{z}^{1}+\cdots+z^{k} \bar{z}^{k}\right)} .\right.
$$

Put $\Omega=d d^{c} \log |z|^{2}$ on $C^{k}-\{0\}$. Then we have the following

LEMMA 3.5. Let $\omega_{\alpha \beta}$ be as before $(1 \leq \alpha, \beta \leq k)$. Then it follows that

$$
\begin{array}{cc}
\tilde{\alpha} & \tilde{\alpha} \\
\vee & \vee \\
\sum_{\tilde{\alpha}=2}^{k} \omega_{21} \wedge \cdots \wedge \omega_{k 1} \wedge \bar{\omega}_{21} \wedge \cdots \wedge \bar{\omega}_{k 1}=\text { const. } \Omega^{k-2},
\end{array}
$$

where $\Omega^{k-2}=\Omega \overbrace{\wedge \cdots \wedge}^{k-2 \text { times }} \Omega$.

Proof. Noting that $\omega_{\alpha \beta}=\sum_{\gamma} d a_{\alpha_{\gamma}} b_{r \beta}$, we get

$$
\sum_{\tilde{\alpha}} \omega_{\tilde{\alpha} 1} \wedge \bar{\omega}_{\tilde{\alpha} 1}=\sum_{\beta} d a_{1 \beta} \wedge d \bar{a}_{1 \beta} .
$$

But from the representations of $a_{1 \beta}$ with respect to $\left\{z^{1}, \cdots, z^{k}\right\}$, we see that 


$$
\begin{aligned}
\sum_{\tilde{\alpha}} \omega_{\tilde{\alpha} 1} \wedge \bar{\omega}_{\tilde{\alpha} 1} & =\sum_{\beta} d\left(\frac{z^{\beta}}{|z|}\right) \wedge d\left(\frac{\bar{z}^{\beta}}{|z|}\right) \\
& =-\sum_{\beta} d\left(d\left(\frac{z^{\beta}}{|z|}\right) \cdot \frac{\bar{z}^{\beta}}{z}\right) \\
& =\text { const. } \Omega .
\end{aligned}
$$

From this fact lemma is proved.

Q.E.D.

Moreover we have the next lemma concerned with the above one.

LEMMA 3.6. Let $S\left(C^{k}\right)$ be the unit sphere of $\boldsymbol{C}^{k}$ and $j_{S}: S\left(C^{k}\right) \rightarrow C^{k}$ $-\{0\}$ be the inclusion. Furthermore let $\alpha=\left(\alpha_{1}, \cdots, \alpha_{t}\right)$ and $\beta=\left(\beta_{1}, \cdots\right.$, $\beta_{t}$ ) be t-multiple indices for any positive integer $t$ such that $1 \leq \alpha_{i}, \beta_{i} \leq k$ $(i=1, \cdots, t)$. Then we find

$$
j_{S}^{*}\left(\Omega^{k-1}\right) \wedge d^{c}\left(\frac{z^{\alpha} \bar{z}^{\beta}}{|z|^{2 t}}\right)=0
$$

where $z^{\alpha}=z^{\alpha_{1}} \cdots z^{\alpha_{t}}$ and $\bar{z}^{\beta}=\bar{z}^{\beta_{1}} \cdots \bar{z}^{\beta_{t}}$.

Proof. It suffices to prove (3.16) in case of $t=1$, that is $1 \leq \alpha$, $\beta \leq k$. Clearly we get

$$
j_{S}^{*}(\Omega)=\sum_{\alpha, \beta=1}^{k}\left(\delta_{\alpha \beta}-\bar{z}^{\alpha} z^{\beta}\right) d z^{\alpha} \wedge d \bar{z}^{\beta},
$$

where $\delta_{\alpha \beta}$ is Kronecker index.

Here let us put

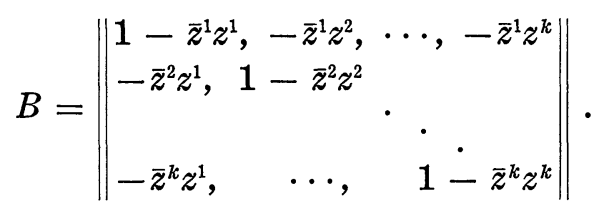

Then

$$
\begin{array}{ccc}
\alpha & \beta \\
j_{S}^{*}\left(\Omega^{k-1}\right)=\text { const. } \sum_{\alpha, \beta} \Delta_{\alpha \beta}(B) d z^{1} \wedge \cdots \wedge d z^{k} \wedge d \bar{z}^{1} \wedge \cdots \wedge d \bar{z}^{k}, & \vee
\end{array}
$$

where $\Delta_{\alpha \beta}(B)$ denotes the $(\alpha, \beta)$-cofactor of $B$. Now we find

$$
d^{\prime}\left(\frac{z^{\alpha} \bar{z}^{\beta}}{|z|^{2}}\right)=\frac{d z^{\alpha} \bar{z}^{\beta}}{|z|^{2}}-\frac{z^{\alpha} \bar{z}^{\beta}}{|z|^{4}}\left(\sum_{r} \bar{z}^{\gamma} d z^{r}\right) .
$$


And so it follows that

$$
\begin{aligned}
j_{S}^{*}\left(\Omega^{k-1}\right. & \wedge d^{\prime}\left(\frac{z^{\alpha} \bar{z}^{\beta}}{|z|^{2}}\right) \\
= & \text { const. }\left\{\bar{z}^{\beta} \sum_{\gamma}\left((-1)^{\alpha-1} \Delta_{\alpha \gamma}(B)-\sum_{\delta}(-1)^{\delta-1} z^{\alpha} \bar{z}^{\delta} \Delta_{\delta_{r}}(B)\right)\right. \\
\gamma & \vee \\
& \left.\times d z^{1} \wedge \cdots \wedge d z^{k} \wedge d \bar{z}^{1} \wedge \cdots \wedge d \bar{z}^{k}\right\}
\end{aligned}
$$

Fixing $r$ in the right hand side of this equation, we obtain

$$
\begin{aligned}
(-1)^{\alpha-1} & \left(\Delta_{\alpha \gamma}(B)-\sum_{\delta}(-1)^{\delta+\alpha} z^{\alpha} \bar{z}^{\delta} \Delta_{\delta_{\gamma}}(B)\right) \\
& =(-1)^{\alpha-1}\left\{\sum_{\delta}(-1)^{\alpha+\delta}\left(\delta_{\alpha, \delta}-z^{\alpha} \bar{z}^{\delta}\right) \Delta_{\delta_{\gamma}}(B)\right\} \\
& = \begin{cases}\operatorname{det}(B)=1-|z|^{2}, & \text { if } \alpha=\gamma \\
0, & \text { otherwise. }\end{cases}
\end{aligned}
$$

But as $|z|=1$ on $S\left(C^{k}\right)$, the right hand side of (3.17) equals to zero for any $\gamma$, so that

$$
j_{S}^{*}\left(d^{\prime}\left(\frac{z^{\alpha} \bar{z}^{\beta}}{|z|^{2}}\right) \wedge \Omega^{k-1}\right)=0 .
$$

Similarly we can prove

$$
j_{S}^{*}\left(d^{\prime \prime}\left(\frac{z^{\alpha} \bar{z}^{\beta}}{|z|^{2}}\right) \wedge \Omega^{k-1}\right)=0
$$

Thus from $d^{c}=i\left(d^{\prime \prime}-d^{\prime}\right)$, (3.16) follows.

Q.E.D.

Now we are in a position to prove (3.7)' For simplicity, set

$$
\mathrm{I}=d^{c} \log \left(\prod_{\mathrm{I}}^{\#} N_{\mathrm{I}}\right)(\Delta \circ \iota) \wedge \lim _{s \rightarrow-\infty} d^{c} \int_{s}^{0} b_{n-1}^{n-1}\left(\left(K\left[\iota_{Q}^{\#}(E), D_{t}^{Q}\right] ; P_{\mathrm{I}}^{Q}\right)\right) d s .
$$

By the definition of the canonical section $\Delta$ (see the above of (3.9)),

$$
\log \left(\prod_{I}^{\#} N_{\mathrm{I}}\right) \Delta \circ \iota\left(x, z^{1}, \cdots, z^{k}\right)=\log |z|^{2},
$$

and so we find from (3.15) and Lemma 3.5 that

$$
I=\sum_{\substack{t \\ \alpha=\left(\alpha_{1}, \ldots, \alpha_{t}\right) \\ \beta=\left(\beta_{1}, \cdots, \beta_{t}\right)}} \psi_{\alpha \beta, t} d^{c} \log |z|^{2} \wedge d d^{c}\left(\Omega^{k-1} \cdot \frac{z^{\alpha} \bar{z}^{\beta}}{|z|^{2 t}}\right)+\Phi_{2 k-2},
$$


where the $\Phi_{\alpha \beta, t}$ are functions on the base space $\tilde{U}(\subset V)$, and $F\left(\Phi_{2 k-2}\right)$ $\leq 2 k-2$. Further using $d^{\prime} \Omega=d^{\prime \prime} \Omega=0$, it follows from (3.18) that

$$
\begin{aligned}
\mathrm{I}= & \sum_{\substack{t \\
\alpha, \beta}} \psi_{\alpha \beta, t}\left\{-d\left(d^{c} \log |z|^{2} \wedge \Omega^{k-2} \wedge d^{c}\left(\frac{z^{\alpha} \bar{z}^{\beta}}{|z|^{2 t}}\right)\right)\right. \\
& \left.+\Omega^{k-1} \wedge d^{c}\left(\frac{z^{\alpha} \bar{z}^{\beta}}{|z|^{2 t}}\right)\right\}+\Phi_{2 k-2}
\end{aligned}
$$

As before let $\iota_{S}$ be the inclusion of $S\left(E_{\mathrm{I}}\right)$ into $\left(E_{\mathrm{I}}\right)_{0}$. Then by (3.16),

$$
\iota_{S}^{*} \mathrm{I}=\sum_{\substack{t \\ \alpha, \beta}} \iota_{S}^{*}\left(-\psi_{\alpha \beta, t}\right) d \iota_{S}^{*}\left(d^{c} \log |z|^{2} \wedge \Omega^{k-2} \wedge d^{c}\left(\frac{z^{\alpha} \bar{z}^{\beta}}{|z|^{2 t}}\right)\right)+\iota_{S}^{*} \Phi_{2 k-2} .
$$

And noting that $\psi_{\alpha \beta, t}$ is constant on each fibre of $\Pi_{\mathrm{I}}:\left(E_{\mathrm{I}}\right)_{0} \rightarrow V$, we see that the fibre integral $P_{*}\left(\iota_{S}^{*} I\right)$ of $\iota_{S}^{*} I$ is zero;

$$
P_{*}(\iota * \mathrm{I})=0 .
$$

Thus the fibre integral of the first term in the right hand side of (3.7) is equal to zero and we can prove similarly that other terms are so.

The above facts show Proposition 3.3, and so our main lemma is proved as stated after this Proposition.

\section{§4. Proof of Theorem 1.9}

As in the statement of Theorem 1.9 let $V$ and $W$ be compact connected complex manifolds of $\operatorname{dim}_{C} V=n$ and $\operatorname{dim}_{C} W=k$. Moreover let $f$ be an (IF)-holomorphic map with rank $f \geqq k-1$, and let us denote by $\Sigma$ the singular set of $f$ (cf. Definition 1.1).

Then in terms of Proposition 1.8 we take an $f$-form $\omega$ on $W$ (Definition 1.7) such that

i) $\omega$ is a $(1,0)$-type differential form on $W$

ii) zeros of $\omega$ is isolated, say, $\left\{q_{1}, \cdots, q_{m}\right\}$ and $f(\Sigma) \cap \operatorname{zeros}(\omega)=\phi$.

iii) $\Sigma \cap\left(\right.$ zeros of $\left.f^{*} \omega\right)=\left\{P_{1}, \cdots, P_{\ell}\right\}$, where the $P_{j}$ are generic points of $\Sigma$.

Let $N$ be a hermitian norm on the holomorphic cotangent bundle $T^{*} V$ of $V$ and let $C_{n}\left(T^{*} V\right)$ be the $n$-th Chern form defined by $N$ as in $\S 2$. First it is well-known that

$$
\chi(V)=(-1)^{n} \int_{V} C_{n}\left(T^{*} V\right)
$$


where $\chi(V)$ is the Euler number of $V$.

On the other hand, as $\operatorname{rank} f \geqq k-1$, it is easy to see, from the above conditions i), ii) and iii), that $f^{*}(\omega)$ becomes the smooth-section of $\left(T^{*} V\right)_{0}=\left\{v d^{*} V ; v \neq 0\right\}$, over $V-\left\{P_{1}, \cdots, P_{\ell}\right\} \cup \bigcup_{j=1}^{m} f^{-k}\left(q_{j}\right)$. Moreover since $q_{j}$ is the regular values of $f$ by ii), $(j=1, \cdots, m)$, we can choose the $\varepsilon$-balls $U_{\varepsilon}\left(p_{j}\right)$ with center $q_{j}$ in $W$ such that for each $j$

$$
f: f^{-1}\left(U_{\varepsilon}\left(q_{j}\right)\right) \rightarrow U_{\varepsilon}\left(q_{j}\right) \quad \text { is a fibre bundle, }
$$

and

$$
U_{\varepsilon}\left(q_{i}\right) \cap U_{\varepsilon}\left(q_{j}\right)=\phi \quad \text { for } i \neq j .
$$

Take also the $\varepsilon$-ball $V_{\varepsilon}\left(p_{j}\right)$ for each $p_{j}$ such that

$$
V_{\varepsilon}\left(p_{i}\right) \cap V_{\varepsilon}\left(p_{j}\right)=\phi \quad \text { for } i \neq j
$$

and

$$
V_{\iota}\left(p_{j}\right) \cap F^{-1}\left(U_{\iota}\left(q_{i}\right)\right)=\phi \quad \text { for } 1 \leq j \leq \ell, 1 \leq i \leq m .
$$

Here put

$$
V_{s}=V-\bigcup_{j=1}^{\ell} V_{\epsilon}\left(p_{j}\right)-\bigcup_{j=1}^{m} f^{-1}\left(U_{s}\left(q_{j}\right)\right) .
$$

One remarks that $f$ is onto, because $f$ is flat and both of $V$ and $W$ are connected compact manifold. Then if we write $\eta_{n}\left(T^{*} V\right)$ the boundary form of $T^{*} V$ with the norm $N$ (Definition 2.4), we have from (2.8) and Stokes' formula,

$$
\begin{aligned}
\int_{V} C_{n}\left(T^{*} V\right)= & \lim _{\varepsilon \rightarrow 0} \int_{V_{\varepsilon}} C_{n}\left(T^{*} V\right) \\
= & -\sum_{j=1}^{m} \lim _{\varepsilon \rightarrow 0} \int_{\partial f^{-1}\left(U_{\varepsilon}\left(q_{j}\right)\right)}\left(f^{*} \omega\right) \eta_{n}\left(T^{*} V\right) \\
& +\sum_{j=1}^{\ell} \lim _{\varepsilon \rightarrow 0} \int_{\partial U_{\varepsilon}\left(p_{j}\right)}\left(f^{*} \omega\right)^{*} \eta_{n}\left(T^{*} V\right) .
\end{aligned}
$$

We shall actually compute in two parts (A) and (B) each term in the last right hand side of (4.2).

$$
\text { (A) Calculation of } \lim _{\varepsilon \rightarrow 0} \int_{\partial f^{-1}\left(U_{\varepsilon}\left(q_{j}\right)\right)}\left(f^{*} \omega\right)^{*} \eta_{n}\left(T^{*} V\right) \text {. }
$$

For simplicity set $q=q_{j}$, and take a sufficiently small $\varepsilon_{0}$ such that $f: f^{-1}\left(U_{s_{0}}(q)\right) \rightarrow U_{s_{0}}(q)$ is a fibre bundle. Let $\varepsilon_{0}$ be fixed. Here we put 


$$
V_{0}=f^{-1}\left(U_{\varepsilon_{0}}(q)\right) \text { and } W_{0}=U_{s_{0}}(q),
$$

and so $\left.f\right|_{V_{0}}: V_{0} \rightarrow W_{0}$ is the fibre bundle. Recall that $T(f): f^{\sharp}\left(T^{*} W\right) \rightarrow T^{*} V$ is the bundle map defined by

$$
T(f)(p, v)=f_{p}^{*} v \quad \text { for }(p, v) \in f^{\sharp}\left(T^{*} W\right) .
$$

Then we clearly obtain the following exact sequence of holomorphic vector bundles over $V_{0}$;

$$
0 \longrightarrow f^{*}\left(T^{*} W_{0}\right) \stackrel{T(f)}{\longrightarrow} T^{*} V_{0} \longrightarrow T^{*} V_{0} / T(f)\left(f^{*}\left(T^{*} W_{0}\right)\right) \longrightarrow 0 \text {. }
$$

Put

$$
Q\left(V_{0}, W_{0}\right)=T^{*} V_{0} / T(f)\left(f^{\sharp}\left(T^{*} W_{0}\right)\right) .
$$

Since $T^{*} V_{0}$ has naturally the norm $N$, we can apply the above exact sequence to Main lemma in $\S 3$, so that on $\left(f^{\sharp}\left(T^{*} W_{0}\right)\right)_{0}$,

$$
T(f)^{*} \eta_{n}\left(T^{*} V_{0}\right)=\eta_{k}\left(f^{\sharp}\left(T^{*} W_{0}\right)\right) \pi_{1}^{*} C_{n-k}\left(Q\left(V_{0}, W_{0}\right)\right)+\pi_{I}^{*}(\xi)+d \eta,
$$

where $\pi_{\mathrm{I}}$ is the natural projection of $\left(f^{\sharp}\left(T^{*} W_{0}\right)\right)_{0}$ onto $V_{0}$. Let $f^{\sharp}(\omega)$ be the cross-section of $f^{\sharp}\left(T^{*} W\right)$ defined by

$$
f^{\sharp}(\omega)(p)=\left(p, \omega_{f(p)}\right) \quad \text { for } p \in V .
$$

Hence noting that $T(f) f^{\sharp}(\omega)=f^{*} \omega$, we find from (4.3),

$$
\begin{aligned}
\left(f^{*} \omega\right)^{*} \eta_{n}\left(T^{*} V_{0}\right)= & \left(f^{\sharp} \omega\right)^{*} \eta_{k}\left(f ^ { \sharp } ( T ^ { * } W _ { 0 } ) \pi _ { \mathrm { I } } ^ { * } C _ { n - k } \left(Q\left(V_{0}, W_{0}\right)\right.\right. \\
& +\pi_{\mathrm{I}}^{*}(\xi)+d\left(f^{\sharp}(\omega)\right)^{*} \eta .
\end{aligned}
$$

Now put

$$
\mathrm{I}=\lim _{\varepsilon \rightarrow 0} \int_{\partial f^{-1}\left(U_{\varepsilon}(q)\right)}\left(f^{*} \omega\right)^{*} \eta_{n}\left(T^{*} V\right)
$$

First we remark that for any $\varepsilon<\varepsilon_{0}$.

$$
\int_{\partial f^{-1}\left(U_{\varepsilon}(q)\right)}\left(f^{*} \omega\right)^{*} \eta_{n}\left(T^{*} V\right)=\int_{\partial f^{-1}\left(U_{\varepsilon}(q)\right)}\left(f^{*} \omega\right)^{*} \eta_{n}\left(T^{*} V_{0}\right),
$$

because of $\left(f^{*} \omega\right)\left(f^{-1}\left(U_{\varepsilon}(q)\right)\right) \subset T^{*} V_{0}$. Then it follows from (4.4) and (4.5) that

(4.6) $\quad \mathrm{I}=\lim _{\epsilon \rightarrow 0} \int_{\partial f^{-1}\left(U_{\epsilon}(q)\right)}\left(f^{\sharp}(\omega)\right)^{*}\left\{\eta_{k}\left(f^{\sharp}\left(T^{*} W_{0}\right)\right) \pi_{\mathrm{I}}^{*} C_{n-k}\left(Q\left(V_{0}, W_{0}\right)\right)+\pi_{\mathrm{I}}^{*} \xi\right\}$. 
First of all we shall show

$$
\lim _{\epsilon \rightarrow 0} \int_{\partial f^{-1}\left(U_{\varepsilon}(q)\right)}\left(f^{\#}(\omega)\right) * \pi_{I}^{*} \xi=0 .
$$

In fact as $f^{-1}\left(U_{\bullet}(q)\right)$ is diffeomorphic to $U_{\bullet}(q) \times f^{-1}(q)$ and $f^{-1}(q)$ is compact, we see

$$
\partial f^{-1}\left(U_{\varepsilon}(q)\right) \cong \partial U_{s}(q) \times f^{-1}(q) \quad \text { (diffeo.) } .
$$

Here noting that $f^{-1}(q)$ is the compact manifold of real dimension $2(n-k)$ $(k \geq 1)$ and that $\xi$ is the $(2 n-1)$-form on $V_{0}$, we can prove (4.7) by virtue of $\left(f^{\sharp}(\omega)\right)^{*} \cdot \pi_{I}^{*}=$ identity.

Next for the purpose of computations of

$$
\lim _{\varepsilon \rightarrow 0} \int_{\partial f-1\left(U_{\varepsilon}(q)\right)}\left(f^{\sharp} \omega\right)^{*} \eta_{k}\left(f^{\sharp}\left(T^{*} W_{0}\right)\right) \wedge C_{n-k}\left(Q\left(V_{0}, W_{0}\right)\right),
$$

let $N_{W}$ be a hermitian norm on $T^{*} W$ and let $f^{\sharp}\left(N_{W}\right)$ be the induced norm on $f^{\sharp}\left(T^{*} W\right)$ by $f$ and $N_{W}$. We denote by $\eta_{k}\left(f^{\sharp}\left(T^{*} W\right), f^{\sharp}\left(N_{W}\right)\right)$ the boundary form of $f^{\sharp}\left(T^{*} W\right)$ associated with the norm $f^{\sharp}\left(N_{W}\right)$. Then the naturality of boundary forms [6] shows that

$$
\eta_{k}\left(f^{\sharp}\left(T^{*} W\right), f^{\sharp}\left(N_{W}\right)\right)=\tilde{f}^{*}\left(\eta_{k}\left(T^{*} W, N_{W}\right)\right),
$$

where $\tilde{f}: f^{\sharp}\left(T^{*} W\right) \rightarrow T^{*} W$ is the map defined by

$$
\tilde{f}(p, v)=v \quad \text { for }(p, v) \in f^{\sharp}\left(T^{*} W\right) .
$$

Furthermore let $N^{\#}$ be any hermitian norm on $f^{\sharp}\left(T^{*} W\right)$. Then we have the following homotopy lemma of boundary forms.

LEMMA 4.1. With the above notations,

$$
\begin{aligned}
& \eta_{k}\left(f^{\sharp}\left(T^{*} W\right), N^{\sharp}\right)-\eta_{k}\left(f^{\sharp}\left(T^{*} W\right), f^{\sharp}\left(N_{W}\right)\right) \\
& \quad=\pi_{\mathrm{I}}^{*}\left(\xi_{k}\right)+d \eta_{k}, \quad \text { on }\left(f^{\sharp}\left(T^{*} W\right)\right)_{0} .
\end{aligned}
$$

Proof. It is easy to see from (2.8) and the homotopy lemma of Chern forms [1] that for some $\xi_{k} \in A^{2 k-1}(V)$,

$$
d \eta_{k}\left(f^{\sharp}\left(T^{*} W\right), N^{\sharp}\right)=d \eta_{k}\left(f^{\sharp}\left(T^{*} W\right), f^{\sharp}\left(N_{W}\right)\right)=\pi_{I}^{*}\left(\xi_{k}\right) .
$$

But since the fibre integral of any boundary form of a holomorphic vector bundle is equal to -1 , we can prove (4.9), combining (4.10) with Lemma 3.2.

Q.E.D. 
Applying here $\eta_{k}\left(f^{*}\left(T^{*} W\right)\right)$ to (4.9), we obtain from (4.9) and $\tilde{f} \circ f^{\sharp}(\omega)$ $=\omega \circ f$,

$$
\begin{aligned}
& \left(f^{\sharp}(\omega)\right)^{*} \eta_{k}\left(f^{\sharp}\left(T^{*} W\right)\right) \\
& \quad=f^{*} \omega^{*} \eta_{k}\left(T^{*} W, N_{W}\right)+\left(f^{\sharp}(\omega)\right) * \pi_{I}^{*} \xi_{k}+d f^{\sharp}(\omega)^{*} \eta_{k} .
\end{aligned}
$$

Let us put

$$
\mathrm{I}_{1}=\lim _{\varepsilon \rightarrow 0} \int_{\partial f^{-1}\left(U_{\varepsilon}(q)\right)}\left(f^{\sharp} \omega\right)^{*} \eta_{k}\left(f^{\sharp}\left(T^{*} W_{0}\right)\right) \wedge C_{n-k}\left(Q\left(V_{0}, W_{0}\right)\right) .
$$

Then using $d C_{n-k}\left(Q\left(V_{0}, W_{0}\right)\right)=0$, we have from $(4.9)^{\prime}$

$$
\left.\mathrm{I}_{1}=\lim _{\boldsymbol{s} \rightarrow 0} \int_{\partial f^{-1}\left(U_{s}(q)\right)} f^{*} \circ \omega^{*} \eta_{k}\left(T^{*} W_{0}, N_{W}\right) \wedge C_{n-k}\left(V_{0}, W_{0}\right)\right),
$$

so that from $f^{-1}\left(U_{\odot}(q)\right) \cong U_{s}(q) \times f^{-1}(q)$,

$$
\mathrm{I}_{1}=\lim _{\varepsilon \rightarrow 0} \int_{\partial U_{\varepsilon}(q) \ni q^{\prime}}\left(\int_{f^{-1}\left(q^{\prime}\right)} C_{n-k}\left(Q\left(V_{0}, W_{0}\right)\right)\right) \cdot\left(\omega^{*} \eta_{k}\left(T^{*} W_{0}, N_{W}\right)\right)_{q^{\prime}} \cdot
$$

Here we have the following

LEMMA 4.2. For any $q^{\prime} \in \partial U_{s}(q)$, we get

$$
\int_{f-1\left(q^{\prime}\right)} C_{n-k}\left(Q\left(V_{0}, W_{0}\right)\right)=(-1)^{n-k} \chi(F),
$$

where $F$ is a general fibre of $f: V \rightarrow W$.

Proof. Let $i_{q^{\prime}}$ be the inclusion of $f^{-1}\left(q^{\prime}\right)$ into $V$. Then as $f$ is fibre map on $f^{-1}\left(U_{\varepsilon_{0}}(q)\right)\left(\varepsilon<\varepsilon_{0}\right)$ and $Q\left(V_{0}, W_{0}\right)=T^{*} V_{0} / T(f)\left(f^{\sharp}\left(T^{*} W_{0}\right)\right)$, it is clear that $\iota_{q^{\prime}}^{\prime} Q\left(V_{0}, W_{0}\right)$ is isomorphic to $T^{*}\left(f^{-1}\left(q^{\prime}\right)\right)$. Therefore we find

$$
\hat{C}_{n-k}\left(\iota_{q}^{\sharp}, Q\left(V_{0}, W_{0}\right)=\hat{C}_{n-k}\left(T^{*}\left(f^{-1}\left(q^{\prime}\right)\right),\right.\right.
$$

where ${ }^{\wedge}$ represents the cohomology class. And also by [2] it follows that

$$
\hat{C}_{n-k}\left(\iota_{q^{\prime}}^{\sharp} Q\left(V_{0}, W_{0}\right)\right)=\iota_{q^{\prime}}^{*} \hat{C}_{n-k}\left(Q\left(V_{0}, W_{0}\right)\right),
$$

so that we have

$$
\int_{f^{-1}\left(q^{\prime}\right)} C_{n-k}\left(Q\left(V_{0}, W_{0}\right)\right)=(-1)^{n-k} \chi\left(f^{-1}\left(q^{\prime}\right)\right) .
$$

On the other hand recall that $\Sigma$ is the singular set of $f$. Then $\left.f\right|_{(V-\Sigma)}:(V-\Sigma) \rightarrow(W-f(\Sigma))$ is the fibre bundle and $f(\Sigma)$ is the analytic set of $W$ with $\operatorname{dim}_{C} f(\Sigma) \leqq k-1$. Thus $(W-f(\Sigma)$ ) being connected, it 
follows that $f^{-1}\left(q_{1}\right)$ is diffeomorphic to $f^{-1}\left(q_{2}\right)$ for any $q_{i} \in W-f(\Sigma)$, $i=1,2$. Hence (4.12) is trivial from (4.13).

Q.E.D.

Moreover we know in virtue of [1] that

$$
\lim _{\varepsilon \rightarrow 0} \int_{\partial U_{\varepsilon}(q)} \omega^{*} \eta_{k}\left(T^{*} W_{0}, N_{W}\right)=-\mathrm{I}_{q}(\omega),
$$

where $\mathrm{I}_{q}(\omega)$ is the degree of zeros of $\omega$ at $q$. From (4.11), (4.12) and (4.14) it follows

$$
\mathrm{I}_{1}=(-1)^{n-k} \chi(F) \mathrm{I}_{q}(\omega)
$$

Thus

$$
\begin{gathered}
\sum_{j=1}^{m} \lim _{e \rightarrow 0} \int_{\partial f^{-1}\left(U_{s}\left(q_{j}\right)\right)}\left(f^{*} \omega\right)^{*} \eta_{n}\left(T^{*} V\right) \\
\quad=(-1)^{n-k+1} \chi(F)\left\{\sum_{j=1}^{m} \mathrm{I}_{q_{j}}(\omega)\right\} \\
=(-1)^{n+1} \chi(F) \chi(W),
\end{gathered}
$$

here we used $\chi(W)=(-1)^{k} \int_{W} C_{k}\left(T^{*} W\right)=(-1)^{k}\left\{\sum_{j=1}^{m} I_{q_{j}}(\omega)\right\}$.

(B) Calculation of $\lim _{\epsilon \rightarrow 0} \int_{\partial U_{\epsilon}\left(p_{j}\right)}\left(f^{*} \omega\right)^{*} \eta_{n}\left(T^{*} V\right)$.

Let us put $p=p_{j}$ and $f(p)=q$. Remember that $p$ is a generic point in the singular set $\Sigma$ of $f$. As $\operatorname{rank}_{p} f=k-1$, we are able to choose a holomorphic chart $\left(\left\{z^{j}\right\}_{j=1}^{k}, U_{q}\right)$ at $q$ with $z^{j}(q)=0,(j=1, \ldots, k)$ such that $\left(d z^{i} \circ f\right)_{p}, \cdots$, and $\left(d z^{k-1} \circ f\right)_{p}$ are linearly independent, and so take a holomorphic chart $\left(\left\{w^{j}\right\}_{j=1}^{n}, U_{p}\right)$ at $p$ such that $w^{j}=z^{j} \circ f(1 \leq j \leq k-1)$ and $w^{j}(p)=0(1 \leq j \leq n)$. Suppose that $f\left(U_{p}\right) \subset U_{q}$ and that $\omega$ never vanishes on $U_{q}$ because of $\omega_{q} \neq 0$. Now let

$$
\omega=\sum_{j=1}^{k} a_{j} d z^{j} \quad \text { on } U_{q} .
$$

Then from the choice of holomorphic coordinates $\left\{z^{i}\right\}_{i=1}^{k}$ and $\left\{w^{j}\right\}_{j=1}^{n}$ it is clear that on $U_{p}$

$$
f^{*} \omega=\sum_{i=1}^{k-1}\left\{a_{i} \circ f+\left(a_{k} \circ f\right) \frac{\partial f^{k}}{\partial w^{i}}\right\} d w^{i}+\sum_{j=k}^{n}\left(a_{k} \circ f\right) \frac{\partial f^{k}}{\partial w^{\alpha}} d w^{\alpha},
$$

where $f^{k}=z^{k} \circ f$.

Here let

$$
\mathrm{I}_{2}=\lim _{\iota \rightarrow 0} \int_{\partial U_{\epsilon}(p)}\left(f^{*} \omega\right)^{*} \eta_{n}\left(T^{*} V\right)
$$


Then we know already that

$$
\mathrm{I}_{2}=-\mathrm{I}_{p}\left(f^{*} \omega\right)
$$

To compute $\mathrm{I}_{p}\left(f^{*} \omega\right)$ let us first examine local properties of the singular set $\Sigma$ about $p$. From $w^{j}=z^{j} \circ f(1 \leq j \leq k-1)$ and rank $f \geqq k-1$, we observe

$$
U_{p} \cap \Sigma=\left\{\frac{\partial f^{k}}{\partial w^{k}}=0, \cdots, \frac{\partial f^{k}}{\partial w^{n}}=0\right\}
$$

Set $\Sigma_{p}=U_{p} \cap \Sigma$. Since $p$ is generic, we can assume that $\Sigma_{p}$ is a complex manifold of dimension $n$. Therefore there exists an $(n-k)$-dimensional complex submanifold of $V$ such that $\Sigma_{p} \cap \Sigma_{p}^{\perp}=\{p\}$ (transversal at $p$ ) and $\partial \Sigma_{p}^{\perp} \cong S^{2 k-1}$, called a complemental submanifold of $\Sigma_{p}$ at $p$.

At first we see that if $U_{p}$ is a sufficiently small neighborhood of $p$,

$$
\left(a_{k} \circ f\right)\left(p^{\prime}\right) \neq 0 \quad \text { for any } p^{\prime} \in U_{p} \text {. }
$$

Indeed from $f_{p}^{*} \omega=0$ and (4.17) it follows that

$$
\begin{cases}\left(a_{i} \circ f\right)(p)+a_{k} \circ f(p) \frac{\partial f^{k}}{\partial w^{i}}(P)=0, & (i=1, \cdots, k-1), \\ \left(a_{k} \circ f\right)(p) \frac{\partial f^{k}}{\partial w^{\alpha}}(P)=0 & (\alpha=k, \cdots, n) .\end{cases}
$$

However as $\omega_{q} \neq 0$, it is easy to see that

$$
\left(a_{k} \circ f\right)(p)=a_{k}(q) \neq 0 \text {. }
$$

This means (4.20).

Now let $v=\left(a_{k} \circ f\right)\left(\frac{\partial f^{k}}{\partial w^{k}}, \cdots, \frac{\partial f^{k}}{\partial w^{n}}\right)$ be the holomorphic map of $U_{p}$ into $C^{n-k+1}$, related with (4.17). Then we have by (4.19) and (4.20).

$$
\text { zeros of } v=\Sigma_{p} \text {, }
$$

and so

$$
\text { zeros of } v \mid \Sigma_{p}^{\perp}=\{p\} \text {. }
$$

LEMMA 4.3. Let $v$ be as above and let $\mu_{f}(p)$ be the obstruction number of $f$ at the generic point $p$ (Def. 13). Then we have

$$
\mathrm{I}_{p}\left(\left.v\right|_{\Sigma} \frac{1}{p}\right)=\mu_{f}(p)
$$


Proof. First we shall recall the definition of $\mu_{f}(p)$. Let $\partial f / \partial w$ be the Jacobian matrix of $f$ with respect to the above coorinates $\left\{w^{i}\right\}_{i=1}^{n}$ and $\left\{z^{i}\right\}_{i=1}^{k}$. Then

$$
\frac{\partial f}{\partial w}=\left\|\frac{1_{k-1}}{\frac{\partial f^{k}}{\partial w^{1}}, \cdots, \frac{\partial f^{k}}{\partial w^{k-1}}} \mid \frac{0}{\frac{\partial f^{k}}{\partial w^{k}}, \cdots, \frac{\partial f^{k}}{\partial w^{n}}}\right\| .
$$

And here

$$
\mu_{f}(p)=\int_{\partial \Sigma_{p}}\left(\frac{\partial f}{\partial w}\right)^{*} \eta_{n, k},
$$

where $\hat{\eta}_{n, k}$ is the generator of the $(2 n-k)+1$-th cohomology group $H^{2(n-k)+1}(V(n, k ; C) ; Z)$ of the Stiefel manifold as in $\S 1$.

Now let $\Phi$ be the holomorphic map on $U_{p}$ defined by

$$
\Phi=\left\|\frac{1_{k-1}}{0} \mid \frac{0}{v}\right\| .
$$

Then $\left.\Phi\right|_{\Sigma_{p}\{p\}}$ is the map of $\Sigma_{p}^{\perp}-\{p\}$ into $V(n, k ; C)$. Moreover we find, using $a_{k} \circ f \neq 0$ on $U_{p}$, that $\left.\frac{\partial f}{\partial w}\right|_{\Sigma_{p}^{\frac{1}{p}-\{p\}}}$ is homotopic to $\left.\Phi\right|_{\Sigma_{p}^{\frac{1}{p}}[p\}}$. Therefore we get

$$
\mu_{p}(f)=\int_{\partial \Sigma_{p}^{\perp}} \Phi^{*} \eta_{n, k} .
$$

But in terms of Lemma 3.7 in [6] we have

$$
\int_{\partial \Sigma \frac{1}{p}} \Phi^{*} \eta_{n, k}=\int_{\partial \Sigma \frac{\perp}{p}} v^{*} \eta_{n, 1}=\mathrm{I}_{p}\left(\left.v\right|_{\Sigma \frac{1}{p}}\right) .
$$

These facts show (4.22).

Q.E.D.

Next let $u=\left(a \circ f+\left(a_{k} \circ f\right) \frac{\partial f^{k}}{\partial w^{1}}, \cdots, a_{k-1} \circ f+\left(a_{k} \circ f\right) \frac{\partial f^{k}}{\partial w^{k-1}}\right)$ be the map of $U_{p}$ into $C^{k-1}$ similar with $v$. It follows from (zeros of $\left.f^{*} \omega\right) \cap U_{p}$ $=\{p\}$ and (4.21) that

$$
\text { zeros of }\left.u\right|_{\Sigma_{p}}=\{p\} \text {. }
$$

In order to compare $\mathrm{I}_{p}\left(\left.u\right|_{\Sigma_{p}}\right)$ with the restricted index $\tilde{I}_{p}(\omega)$ of $\omega$ at $p$ (Definition 1.6) let be the projection of $\left.f^{*}\left(T^{*} W\right)\right|_{\Sigma}$ onto $Q(\Sigma)=f^{\sharp}\left(T^{*} W\right) /$ $\operatorname{Ker} T(f)$ as denoted in $\S 1$. Then by (4.16) it is trivial that 


$$
\left.\widetilde{f^{\sharp}(\omega)}\right|_{\Sigma_{p}}=\sum_{i=1}^{k}\left(a_{i} \circ f\right) \widetilde{\left.f^{\sharp}\left(d z^{i}\right)\right|_{\Sigma_{p}}}
$$

LEMMA 4.4. Notations being as above, it follows that

$$
\left.\widetilde{f^{\sharp}(\omega)}\right|_{\Sigma_{p}}=\left.\sum_{j=1}^{k-1}\left(a_{j} \circ f+\left(a_{k} \circ f\right) \frac{\partial f^{k}}{\partial w^{j}}\right) \widetilde{f^{\#}\left(d z^{j}\right)}\right|_{\Sigma_{p}}
$$

and that $\left\{\left.f^{\sharp}\left(d z^{j}\right)\right|_{\Sigma_{p}}\right\}_{j=1}^{k-1}$ is a base of $\left.\left.Q_{k}\right) \Sigma\right)\left.\right|_{\Sigma_{p}}$.

Proof. Clearly $\left\{f^{\sharp}\left(d z^{i}\right)\right\}_{i=1}^{k}$ is the base of $f^{\sharp}\left(T^{*} W\right) \mid U_{p}$. Here let $\theta=\Sigma b_{i} f^{\sharp}\left(d z^{i}\right)$ be any section of $f^{\sharp}\left(T^{*} W\right)$ on $U_{p}$. Then

$$
T(f)(\theta)=\sum_{j=1}^{k-1}\left(b_{j}+b_{k} \frac{\partial f^{k}}{\partial w^{j}}\right) d w^{j}+b_{k}\left(\frac{\partial f^{k}}{\partial w^{k}} d w^{k}+\cdots+\frac{\partial f^{k}}{\partial w^{n}} d w^{n}\right) .
$$

But since $\frac{\partial f^{k}}{\partial w^{\alpha}}=0$ on $\Sigma_{p}(\alpha=k, \cdots, n), T(f)(\theta)=0$ means that $b_{j}=-b_{k}$ $\cdot \frac{\partial f^{k}}{\partial w^{j}}$ on $\Sigma_{p}$ for $j=1, \cdots, k-1$. Thus we see that $\sum_{j=1}^{k-1} \frac{\partial f^{k}}{\partial w^{i}} f^{\sharp}\left(d z^{i}\right)-f^{\sharp}\left(d z^{k}\right)$ is the base of $\operatorname{Ker} T(f) \mid \Sigma_{p}$. Here the second statement in Lemma 4.4 is proved. Moreover as

$$
\widetilde{f^{\#}\left(d z^{k}\right)}=\sum_{j=1}^{k-1} \frac{\partial f^{k}}{\partial w^{j}} \widetilde{f^{\sharp}\left(d z^{j}\right)} \quad \text { on } \Sigma_{p},
$$

we can prove directly (4.26).

Q.E.D.

From the above lemma we obtain the following

COROLLARY 4.5 .

$$
\tilde{I}_{p}(\omega)=I_{p}\left(\left.u\right|_{\Sigma_{p}}\right)
$$

Finally let us put

$$
\Phi=(v, u)
$$

Then it is trivial from (4.17) and (4.18) that

$$
I_{p}(f * \omega)=I_{p}(\Phi) .
$$

Under the above preparations we are able to prove the following Proposition 4.6.

$$
I_{p}\left(f^{*} \omega\right)=\mu_{f}(p) \tilde{I}_{p}(\omega)
$$


Proof. From (4.22), (4.27) and (4.28) it is enough to prove

$$
I_{p}(v, u)=I_{p}\left(\left.v\right|_{\Sigma_{p}}\right) \cdot I_{p}\left(\left.u\right|_{\Sigma} \frac{1}{p}\right)
$$

However as we can assume $U_{p} \cong \Sigma_{p} \times \Sigma_{p}^{\perp}$, we observe from $(4.21)^{\prime}$ and (4.24) that

$$
(v, u) \text { is homotopic to }\left(\left.v\right|_{\Sigma_{p}},\left.u\right|_{\Sigma_{p}}\right) \text {. }
$$

And from elementary facts of Topology we have (4.30).

Q.E.D.

The above proposition shows in terms of (4.18)

$$
\lim _{\epsilon \rightarrow 0} \int_{\partial U_{\varepsilon}(p)}\left(f^{*} \omega\right)^{*} \eta_{n}\left(T^{*} V\right)=-\mu_{f}(p) \tilde{I}_{p}(\omega) .
$$

Now we shall complete the proof of Theorem 1.9. First of all it holds from (4.2), (4.15) and (4.29) that

$$
\int_{V} C_{n}\left(T^{*} V\right)=(-1)^{n} \chi(F) \chi(W)+\sum_{j=1}^{\ell} \mu_{f}\left(p_{j}\right) \tilde{I}_{p_{j}}(\omega),
$$

and so from (4.1) we can prove (1.4) in Theorem 1.9.

\section{§5. Appendix}

In this section we shall prove Proposition 1.8 in $\S 1$. Before proving this fact we review definition in [5]. Let $N$ be a smooth manifold and $T^{R} N$ be the real tangent bundle of $N$.

DEFINITION 5.1. Let $S=\left\{S_{i}\right\}_{i \in I}$ be a partition of $N$, that is, $N=\bigcup_{i \in I} S_{i}\left(S_{i} \cap S_{j}=\phi\right.$ if $\left.i \neq j\right)$. Then the partition $S$ is called a stratification of $N$ when the following conditions are satisfied;

a) $I$ is countable,

b) each $S_{i}$ which is called a strata is a regular submanifold of $N$,

c) if for any non-negative integer $p$ we put

$$
I(p)=\left\{i \in I ; \operatorname{dim} S_{i} \leq p\right\},
$$

then union $\cup_{j \in I(p)} S_{j}$ is closed in $N$.

One notices that by conditions a) and c) $S$ is locally finite.

Let $S=\left\{S_{i}\right\}_{i \in I}$ be a stratification and let $J \subset I$. Put $S_{J}=\left\{S_{i} ; i \in J\right\}$. Then we set

$$
\left|S_{J}\right|=\bigcup_{j \in J} S_{j}
$$


Now let $S$ be a stratification of $N$. Then for any $x \in N, T_{x}(S)$ is defined by

$T_{x} S=$ the tangent space $T_{x} S_{i}$ of a strata $S_{i}$ containing $x$. With this notation we state the following

DEFinition 5.2. Let $E$ be a $C^{\infty}$-vector bundle over $N$, and let $\Gamma_{\infty}(E)$ be the set of all smooth sections of $E$. Suppose that a stratification $S=\left\{S_{i}\right\}_{i \in I}$ of $E$ is given. Then a section $\omega \in \Gamma_{\infty}(E)$ is called transversal to $S$ if and only if

$$
\omega_{*}\left(T_{x} N\right)+T_{\omega(x)} S=T_{\omega(x)} E .
$$

We denote by $\AA_{h}(S)$ the set of all transversal cross-section of $E$.

Now let us return to the proof of Proposition 1.8. Let $V$ and $W$ be compact complex manifolds of $\operatorname{dim}_{c} V=n$ and $\operatorname{dim}_{c} W=k$, and let $f$ be an $(I F)$-holomorphic mapping of $V$ into $W$ with $\operatorname{rank} f \geqq k-1$ as in $\S 1$. When $\tilde{f}$ is the linear map of $f^{*}\left(T^{*} W\right)$ onto $T^{*} W$ defined by $\tilde{f}(x, v)=v$ for any $(x, v) \in f^{\sharp}\left(T^{*} W\right)$, we observe that $\tilde{f}$ is proper, because $V$ is compact. Here let $\Sigma$ be the singular set of $f$. Since $\Sigma$ is the analytic set in $V$ of $\operatorname{dim}_{c} \Sigma=k-1$ and $f$ is of rank $k-1$ on $\Sigma$, the closed subset $\left.\operatorname{Ker} T(f)\right|_{\Sigma}$ of $f^{\sharp}\left(T^{*} W\right)$ becomes the $k$-dimensional analytic set, where $T(f): f^{\sharp}\left(T^{*} W\right) \rightarrow T^{*} V$ is defined as follows; for any $(p, v) \in f^{\sharp}\left(T^{*} W\right), T(f)(p, v)=f_{p}^{*} v$. For simplicity set

$$
L(\Sigma)=\tilde{f}\left(\left.\operatorname{Ker} T(f)\right|_{\Sigma}\right) .
$$

Then as $\tilde{f}$ is proper, we find from the proper mapping theorem ([6]) that $L(\Sigma)$ is the analytic set of $T^{*} W$ such that

$$
\operatorname{dim}_{c} L(\Sigma) \leq k .
$$

The next proposition is due to Whitny [8].

Proposition 5.3. Let $M$ be a complex manifold and $\Sigma^{\prime}$ be an analytic set of $M$. Then $M$ has a stratification $S=\left\{S_{i}\right\}_{i \in I}$ such that $M-\Sigma^{\prime}$ is a strata of $S$ and $\bar{S}_{i}-S_{i} \subset \cup_{j \in I(p)} S_{j}\left(p \leq \operatorname{dim}_{R} S_{i}\right)$ for each $i \in I$. We call stratification in this proposition $\Sigma^{\prime}$-stratification of $N$.

At first we get the following

LEMMA 5.4. All notations are as before. Let $\Sigma_{s}$ be the singular set of $\Sigma$ and let $L\left(\Sigma_{s}\right)=\tilde{f}\left(\left.\operatorname{Ker} T(f)\right|_{\Sigma_{s}}\right)$. Further let $S\left(L\left(\Sigma_{s}\right)\right)$ be a $L\left(\Sigma_{s}\right)$ - 
stratification of $T^{*} W$. Then for any $\omega \in \AA_{\AA}\left(S\left(L\left(\Sigma_{s}\right)\right)\right)$ we obtain

$$
\omega^{-1}\left(L\left(\Sigma_{s}\right)\right)=\phi .
$$

Proof. Assume $q \in \omega^{-1}\left(L\left(\Sigma_{s}\right)\right)$. Then it follows from (5.1) that

$$
\omega_{*}\left(T_{q}^{R} W\right)+T_{\omega(q)}^{R} S_{i}=T_{\omega(q)}^{R}\left(T^{*} W\right),
$$

where $S_{i}(\ni \omega(q))$ is a strata of $S\left(L\left(\Sigma_{s}\right)\right)$.

However $S_{i}$ is contained in $L\left(\Sigma_{s}\right)$ with $\operatorname{dim}_{R} L\left(\Sigma_{s}\right) \leq 2(k-1)$, and so $\operatorname{dim} T_{\omega(q)}^{R} S_{i} \leq 2(k-1)$. This is contrary to (5.5), because $W$ is of real $2 k$-dimension.

Q.E.D.

Secondly it follows the following

LEMMA 5.5. Let $S(L(\Sigma))=\left\{S_{i}\right\}_{i \in I}$ be an $L(\Sigma)$-stratification of $T^{*} W$ and let us denote by $S^{j}(L(\Sigma))$ the set of stratum $S_{i}$ such that $\operatorname{dim}_{R} S_{i} \geq j$.

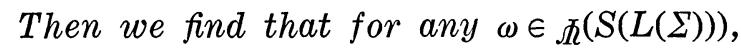

$$
\omega^{-1}\left(\left|S^{2 k}(L(\Sigma))\right|\right) \text { is a finite point set. }
$$

(As $\operatorname{dim}_{R} L(\Sigma) \leq 2 k$ and $S$ is $L(\Sigma)$-stratification, $\left|S^{2 k}(L(\Sigma))\right|$ coincides with $L(\Sigma)$.)

Proof. Take an arbitrary strata $S_{i}$ of real dimension $2 k$. Then $\omega^{-1}\left(S_{i}\right)$ is discrete and without accumulating points. Indeed discreteness is trivial, since $\omega$ is transversal to $S_{i}$. On the other hand suppose $\left\{q_{\alpha}\right\} \subset \omega^{-1}\left(S_{i}\right)$ converges to a point $q_{0}$. Then $\omega\left(q_{p}\right) \in \bar{S}_{i}$ and so $\omega\left(q_{0}\right) \in \bar{S}_{i}$ $-S_{i}$. And from the definition of $L(\Sigma)$-stratification, $\omega\left(q_{0}\right) \in\left|S^{2 k-1}(L(\Sigma))\right|$.

But we can prove similarly as Lemma 5.4 that

$$
\omega^{-1}\left(\left|S^{2 k-1}(L(\Sigma))\right|\right)=\phi .
$$

This show $\omega^{-1}\left(S_{i}\right)$ has not accumlating points. Next recall $S$ is locally finite. Noticing $W$ is compact, we see that $\left\{i ; \omega^{-1}\left(S_{i}\right) \neq \phi\right\}$ is finite. Therefore Lemma 5.5 is proved.

Q.E.D.

Now, $f(\Sigma)$ being the analytic set of $W$ with $\operatorname{dim}_{R} f(\Sigma) \leq 2 k-2$, the zero-section of $\left.T^{*}(W)\right|_{f(\Sigma)}$ is also analytic set. We write for $f(\Sigma)$ this section without confusion. Then with respect to $f(\Sigma)$-stratification $S(f(\Sigma))$ we can also prove that

$$
\left.\omega\right|_{f(\Sigma)} \text { is non-zero for all } \omega \in \epsilon_{\AA}(S(f(\Sigma))) \text {. }
$$

On the other hand we know transversality theorem in [5] that 


$$
D(\Sigma)=\AA^{(S(f(\Sigma)))} \cap \AA_{h}^{\left(S\left(L\left(\Sigma_{s}\right)\right)\right)} \cap \AA_{\AA}(S(L(\Sigma)))
$$

is empty.

Here let us take $\omega \in D(\Sigma)$. Then from the above results it follows that

$$
\omega \text { is non-zero on } f(\Sigma) \text {, }
$$

$$
\omega\left(f\left(\Sigma_{s}\right)\right) \not \subset L\left(\Sigma_{s}\right),
$$

and

$$
\omega^{-1}(L(\Sigma)) \text { is finite, say }\left\{q_{1}, \cdots, q_{m}\right\} \text {, note }\left(q_{i} \in f(\Sigma)\right) \text {. }
$$

Now Proposition 1.8 is trivial. Indeed let $q_{i} \in \omega^{-1}(L(\Sigma))$. This means that $T(f)\left(f^{\sharp}(\omega)(p)\right)=0$ for any $p \in \Sigma \cap f^{-1}\left(q_{i}\right)$. Since from (5.9), $q_{i} \notin f\left(\Sigma_{s}\right)$, we observe that $\Sigma \cap f^{-1}\left(q_{i}\right)$ is in the regular points set of $\Sigma$ and finite, because $f$ has only isolated singularities. Moreover it is clear from (5.8) that $\omega\left(q_{i}\right) \neq 0(i=1, \cdots, m)$. Here $\omega$ satisfy condition i) and ii) of $f$ forms (cf. Definition 1.7). This completes the proof of Proposition 1.8.

\section{REFERENCES}

[1] A. Borel and F. Hirzebruch, Characteristic classes and homogeneous spaces I, Amer. J. Math. 80 (1958), 458-538.

[2] R. Bott and S. S. Chern, Hermitian vector bundles and equidistribution of the zeros of their holomorphic sections, Acta Math. 114 (1965), 71-112.

[ 3 ] R. C. Gunning and H. Rossi, Analytic functions of several complex variables, Prentice-Hall, New Jersey, 1965.

[ 4 ] B. Iversen, Critical points of an algebraic function, Inv. Math. 18 (1971), 210-224.

[5] J. Martinet, Sur les singularites des formes differentielles, Ann. Inst. Fourier, Grenoble 20 (1970), 95-178.

[6] H. Omoto, An integral formula for the Chern form of a hermitian bundles, Nagoya Math. J. 42 (1971), 135-172.

[ 7 ] N. Steenrod, The topology of fiber bundles, Princeton Univ. Press, 1951.

[ 8 ] H. Whiteney, Tangents to an analytic variety, Ann. of Math. 81 (1965), 496-549.

Nagoya University 